\title{
Article \\ A Class of BCI-Algebra and Quasi-Hyper BCI-Algebra
}

\author{
Xiaohong Zhang * and Yudan Du \\ School of Mathematics \& Data Science, Shaanxi University of Science \& Technology, Xi'an 710021, China; \\ 1909010@sust.edu.cn \\ * Correspondence: zhangxiaohong@sust.edu.cn
}

Citation: Zhang, X.; Du, Y. A Class of $B C I$-Algebra and Quasi-Hyper BCI-Algebra. Axioms 2022, 11, 72. https://doi.org/10.3390/

axioms11020072

Academic Editor: Sergey V.

Ludkovsky

Received: 23 December 2021

Accepted: 1 February 2022

Published: 10 February 2022

Publisher's Note: MDPI stays neutral with regard to jurisdictional claims in published maps and institutional affiliations.

Copyright: () 2022 by the authors Licensee MDPI, Basel, Switzerland. This article is an open access article distributed under the terms and conditions of the Creative Commons Attribution (CC BY) license (https:// creativecommons.org/licenses/by/ $4.0 /)$.

\begin{abstract}
In this paper, we study the connection between generalized quasi-left alter $B C I$-algebra and commutative Clifford semigroup by introducing the concept of an adjoint semigroup. We introduce QM-BCI algebra, in which every element is a quasi-minimal element, and prove that each QM-BCI algebra is equivalent to generalized quasi-left alter $B C I$-algebra. Then, we introduce the notion of generalized quasi-left alter-hyper $B C I$-algebra and prove that every generalized quasi-left alter-hyper $B C I$-algebra is a generalized quasi-left alter $B C I$-algebra. Next, we propose a new notion of quasihyper $B C I$ algebra and discuss the relationship among them. Moreover, we study the subalgebras of quasi-hyper $B C I$ algebra and the relationships between $H_{v}$-group and quasi-hyper $B C I$-algebra, hypergroup and quasi-hyper $B C I$-algebra. Finally, we propose the concept of a generalized quasileft alter quasi-hyper $B C I$ algebra and QM-quasi hyper BCI-algebra and discuss the relationships between them and related $B C I$-algebra.
\end{abstract}

Keywords: generalized quasi-left alter $B C I$-algebra; adjoint semigroup; quasi-hyper $B C I$ algebra

\section{Introduction}

In 1966, Japanese mathematicians Imai and Iséki proposed the concepts of $B C K / B C I$ algebra based on logical algebra and the algebraic expression of combinators in combinatorial logic, which are the two kinds of algebraic structures closest to combinatorial logic, fuzzy logic, etc.) [1-5]. From the development of BCI-algebras, we know that $B C I$-algebras can be studied from several aspects:

(1) Find some special classes of BCI-algebra, such as associative BCI-algebra introduced by Qingping $\mathrm{Hu}$ and K. Iséki in 1980 [6]; generalized associative BCI-algebra proposed by Tiande Lei in 1985 [7]; generalized quasi-left alter BCI-algebra by X.H. Zhang in 1992 [8]; the mixed structure of BCI-algebra and semigroup [9].

(2) Starting from the operation of logical algebra, another new operation was derived, which is suitable for associative law. For example, the semigroup structure induced by a BCI-algebra [10]. W.P. Huang studied the adjoint semigroup of generalized associative $B C I$-algebra and proved that the adjoint semigroup of generalized associative $B C I$-algebra is an Abelian group [11]. In 1995, Zhang and Ye first revealed the internal relationship between BZ-algebras and general groups, which can be non-commutative [12].

F. Marty introduced the notion of hyperstructure (also called multialgebra) in 1934 [13], which was widely used in applied sciences (see [14-17]). Naturally, the idea of a hyperstructure is also applied to the study of non-classical logic algebras. Jun et al. introduced hyper BCK-algebra in 2000, and investigated hyper BCK-ideals and some related hyper algebras, such as hyper $K$-algebra and hyper $M V$-algebra (see [18-23]). In 2006, Jun and Borzooei et al. independently proposed the new concept of hyper BCC-algebra; Xin also introduced the definition of hyper BCI-algebra in 2006 and, since then, many research papers on hyper logical algebras have emerged (see [24-31]). In 2021, Y.D. Du and X.H. Zhang introduced the definition of hyper BZ-algebra and discuss the relationships between it and semihypergroups by an adjoint semigroup ([32]). 
In sum, the study of both logical algebra and hyper logic algebra should start from their relationship with classical abstract algebra. Therefore, for this paper, we paid attention to the connection between quasi-hyper $B C I$-algebra and a semihypergroup.

The arrangement of the whole paper is as below. Firstly, we show a number of definitions, properties, and theorems in non-classical logic algebras and related hyper algebraic structures in Section 2. In Section 3, we study the adjoint semigroup of generalized quasi-left alter (hyper) $B C I$-algebra and QM-(hyper) BCI algebra. We obtained some results. In Section 4, we propose a definition of quasi-hyper $B C I$ algebra, discuss some properties, investigate the relationship between quasi-hyper $B C I$-algebra and hyper $B C I$-algebra, weak hyper $B C I$-algebra. Moreover, we study relationships between quasi-hyper $B C I$ algebra, hypergroup and the $H_{v}$-group. Finally, we introduce the concepts of generalized quasi-left alter quasi-hyper $B C I$-algebra and QM-quasi-hyper $B C I$-algebra and discuss the relationships between them and the related $B C I$-algebra.

\section{Preliminaries}

Definition 1 ([33]). Assume that $S$ is a semigroup, $a \in S$. If $\exists x \in S$ s.t. axa $=a$, then $a$ is called regular. $S$ is regular, if each element of $S$ is regular.

Definition 2 ([33]). Assume that $S$ be a semigroup. If $\forall a \in S$, there is a operation $a \mapsto a^{-1}$ on $S$, and it satisfies

$$
\left(a^{-1}\right)^{-1}=a, a a^{-1} a=a, a a^{-1}=a^{-1} a .
$$

Then, it is a completely regular semigroup.

Definition 3 ([33]). Assume that $(S, \mu,-1)$ be a completely regular semigroup. $\forall x, y$ in $S$

$$
\left(x^{-1} x\right)\left(y^{-1} y\right)=\left(y^{-1} y\right)\left(x^{-1} x\right) .
$$

This is a Clifford semigroup.

Definition 4 ([1,2]). Let $\langle X ; *, 0>$ be a type $(2,0)$ algebraic structure, if it satisfies: $\forall x, y, z \in X$,

(BCI1) $((x * z) *(y * z)) *(x * y)=0$;

(BCI2) $(x *(x * y)) * y=0$;

(BCI3) $x * 0=x$;

(BCI4) $x * y=0$ and $y * x=0 \Rightarrow x=y$.

It is a BCI-algebra.

In $B C I$-algebra $\langle X ; *, 0\rangle$, if it satisfies:

(BK) $0 * x=0$, for all $x \in X$;

This is a BCK-algebra.

In $B C K / B C I$-algebra, define $\leq: x \leq y$ iff $x * y=0$.

Theorem 1 ([1]). A type (2,0) algebraic structure $<X ; *, 0>$ is a BCI-algebra iff $\forall x, y, z \in X$, it satisfies:

(BI1) $((x * z) *(y * z)) *(x * y)=0$;

(BI2) $(z * x) * y=(z * y) * x$;

(BI3) $x * x=0$;

(BI4) $x * y=0$ and $y * x=0 \Rightarrow x=y$;

(BI5) $(0 *(0 * x)) * x=0$.

Definition 5 ([6]). BCI-algebra $<X ; *, 0>$ is generalized associative, if $0 *(0 * x)=x, \forall x \in X$.

Theorem 2 ([34]). In any BCI-algebra $\langle X ; *, 0\rangle$, the below conditions are equivalent:if $\forall x, y, z \in X$, 
(1) $X$ is generalized associative;

(2) $x *(0 * y)=y *(0 * x)$;

(3) $0 *(x * y)=y * x$;

(4) $x *(y * z)=z *(y * x)$;

(5) $x *(x * y)=y$;

(6) $(x * y) *(x * z)=z * y$;

(7) $(x * z) *(y * z)=x * y$.

Proposition 1 ([7]). In BCI-algebra $<X ; *, 0>$, the below equations hold:

(1) $0 *(0 *(0 * x))=0 * x, \forall x \in X$;

(2) $0 *(x * y)=(0 * x) *(0 * y), \forall x, y \in X$.

Definition 6 ([7]). The necessary and sufficient condition for a type (2,0) algebraic structure $<X ; *, 0>$ to be a quasi-alter BCK-algebra is that it meets: $\forall x, y \in X$, if $x=y, x * y=0$, otherwise, $x * y=x$.

Definition 7 ([7]). In a BCI-algebra $<X ; *, 0>$, if $\forall x, y \in X$, and $x \neq y$,

$$
x *(x * y)=0 *(0 * y) .
$$

Then, it is said to be a generalized quasi-left alter BCI-algebra.

Theorem 3 ([7]). Assume that $\langle X ; *, 0\rangle$ be a generalized quasi-left alter BCI-algebra. $\forall x \in X$, either $0 * x=0$, or $0 *(0 * x)=x$.

Definition 8. Assume that $\langle X ; *, 0>$ is a BCI-algebra. Then,

$$
B C K(X):=\{m \in X \mid 0 * m=0\}
$$

is a BCK-part of $X$.

Definition 9. Assume that $\langle X ; *, 0\rangle$ is a BCI-algebra. Then,

$$
A G(X):=\{m \in X \mid 0 *(0 * m)=0\}
$$

is a generalized associative part of $X$.

Proposition 2 ([7]). The BCK-part $B(X)$ of the generalized quasi-left alter BCI-algebra $X$ is quasialter $B C K$-subalgebra. Let $G(X)=X-B(X)$ be the BCK-remainder of generalized quasi-left alter $B C I$-algebra $X$. We can see that $G(X) \cup\{0\}$ is generalized associative subalgebra.

Theorem 4 ([7]). Assume that $\langle X ; *, 0\rangle$ is a generalized quasi-left alter BCI-algebra, $B(X)$, $G(X)$ are $B C K$-part and $B C K$-remainder of $X$, respectively. Then:

(1) $x \in B(X), y \in G(X) \cup\{0\}$ imply $x * y=0 * y$;

(2) $x \in G(X) \cup\{0\}, y \in B(X)$ imply $x * y=x$.

Definition 10 ([14]). A hypergroupoid $(H, \circ)$ is a semihypergroup, if $\forall x, y, z \in H$, we have $(x \circ y) \circ z=x \circ(y \circ z)$. That is,

$$
\bigcup_{u \in x \circ y} u \circ z=\bigcup_{v \in y \circ z} x \circ v
$$

In semihypergroup $(H, \circ)$, for all $A, B, C \in P^{*}(H),(A \circ B) \circ C=A \circ(B \circ C)$, where $P^{*}(H)$ represents the non-empty subset of $H$.

Definition 11 ([14]). A semihypergroup $(H, \circ)$ is a hypergroup if $\forall a \in H, a \circ H=H \circ a=H$. 
In a study of hyperstructure, $a \ll b$ represents $0 \in a \circ b$. For each $S, B \subseteq H, S \ll B$ represents that, for all $s \in S$, there is $b \in B$, such that $s \ll b$.

Definition 12 ([18]). Assume that $(H, \circ)$ is a hyper groupoid containing 0 . If it meets these axioms: $\forall x, y, z \in H$,

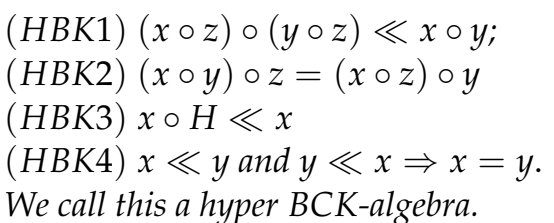

Definition 13 ([26]). Assume that $(H, 0)$ is a hyper groupoid containing 0 . If it meets these axioms: $\forall x, y, z \in H$,

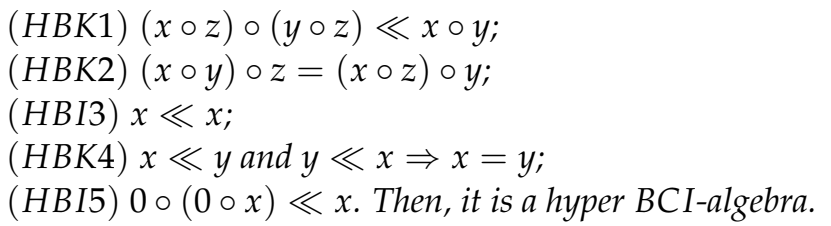

Definition 14 ([24]). Assume that $(H, \circ)$ is a hyper groupoid containing 0 . If it meets these axioms:

$(H B K 1)(x \circ z) \circ(y \circ z) \ll x \circ y, \forall x, y, z \in H$;

$(H B K 2)(x \circ y) \circ z=(x \circ z) \circ y, \forall x, y, z \in H$;

$(H B I 3) x \ll x, \forall x \in H$;

$(H B K 4) x \ll y$ and $y \ll x \Rightarrow x=y, \forall x, y \in H$;

$(H B I 5) 0 \circ(0 \circ x) \ll x, x \neq 0, \forall x \in H$.

Then, it is a weak hyper BCI-algebra.

\section{Generalized Quasi-Left Alter BCI-Algebra}

Firstly, we introduce the adjoint semigroup of $B C I$-algebra and provide some examples about the adjoint semigroup of BCI-algebra. Then, we discuss the relationship between the adjoint semigroup of a generalized quasi-left alter BCI-algebra and commutative Clifford semigroup.

Assume that $\langle X, *, 0\rangle$ is a $B C I$-algebra. $\forall a, x \in X$, denote a map $\rho_{a}$ :

$$
\rho_{a}: X \rightarrow X: x \mapsto x * a .
$$

$\forall a, b \in X$, denote $\rho_{a} \circledast \rho_{b}: X \rightarrow X$ as follows: $\forall x \in X$,

$$
x \mapsto\left(\rho_{a} \circledast \rho_{b}\right)(x)=(x * b) * a .
$$

where $\circledast$ represents the composition operation of mappings.

Theorem 5. Assume that $\langle X, *, 0\rangle$ is a BCI-algebra. Denote $M(X)$ as a set of finite products $\rho_{a_{1}} \circledast \ldots \circledast \rho_{a_{s}}\left(a_{1}, \ldots, a_{s} \in X\right)$, where $\circledast$ represents the composition operation of mappings. Then, $(M(X), \circledast)$ is a monoid and it is commutative.

Proof. $\forall a, b, c \in X$, and $\forall x \in X$, we have

$$
\begin{gathered}
\left(\rho_{a} \circledast \rho_{b}\right) \circledast \rho_{c}(x)=\rho_{a} \circledast \rho_{b}(x * c)=((x * c) * b) * a, \\
\rho_{a} \circledast\left(\rho_{b} \circledast \rho_{c}\right)(x)=\rho_{a} \circledast\left(\rho_{b} \circledast \rho_{c}(x)\right)=\rho_{a}((x * c) * b)=((x * c) * b) * a,
\end{gathered}
$$

Obviously, $\left(\rho_{a} \circledast \rho_{b}\right) \circledast \rho_{c}(x)=\rho_{a} \circledast\left(\rho_{b} \circledast \rho_{c}\right)(x)$. So $(M(X), \circledast)$ satisfies associative law. 
$\forall x \in X, \rho_{a} \in M(X)$,

$$
\begin{aligned}
& \rho_{0} \circledast \rho_{a}(x)=\rho_{0}(x * a)=(x * a) * 0=x * a=\rho_{a}(x), \\
& \rho_{a} \circledast \rho_{a}(x)=\rho_{a}(x * 0)=(x * 0) * a=x * a=\rho_{a}(x) .
\end{aligned}
$$

Then, $\rho_{0}$ is the identity element in $M(X)$. Therefore, $(M(X)$, $\circledast)$ is monoid. $\forall m, n \in X$, and $\forall x \in X$,

$$
\rho_{m} \circledast \rho_{n}(x)=(x * n) * m=(x * m) * n=\rho_{n} \circledast \rho_{m}(x) .
$$

Therefore, $(M(X), \circledast)$ is commutative.

Example 1. Assume that $X=\{0,1,2,3,4,5\}$. This operation on $X$ is shown in Table 1 .

Table 1. BCI-algebra.

\begin{tabular}{lllllll}
\hline$*$ & $\mathbf{0}$ & $\mathbf{1}$ & $\mathbf{2}$ & $\mathbf{3}$ & $\mathbf{4}$ & $\mathbf{5}$ \\
\hline 0 & 0 & 0 & 0 & 3 & 5 & 4 \\
1 & 1 & 0 & 1 & 3 & 5 & 4 \\
2 & 2 & 0 & 0 & 3 & 5 & 4 \\
3 & 3 & 3 & 3 & 0 & 4 & 5 \\
4 & 4 & 4 & 4 & 5 & 0 & 3 \\
5 & 5 & 5 & 5 & 4 & 3 & 0 \\
\hline
\end{tabular}

Clearly, $<X, *, 0>$ is a BCI-algebra .

That is, $\rho_{0}: X \rightarrow X: x \mapsto x * 0$. So $\rho_{0}=\{0,1,2,3,4,5\}$;

$\rho_{1}: X \rightarrow X: x \mapsto x * 1$. So $\rho_{1}=\{0,0,0,3,4,5\}$;

$\rho_{2}: X \rightarrow X: x \mapsto x * 2$. So $\rho_{2}=\{0,1,0,3,4,5\}$;

$\rho_{3}: X \rightarrow X: x \mapsto x * 3$. So $\rho_{3}=\{3,3,3,0,5,4\}$;

$\rho_{4}: X \rightarrow X: x \mapsto x * 4$. So $\rho_{4}=\{5,5,5,4,0,3\}$;

$\rho_{5}: X \rightarrow X: x \mapsto x * 5$. So $\rho_{5}=\{4,4,4,5,3,0\}$.

We could confirm the following:

$\rho_{0} \circledast \rho_{0}=\rho_{0}, \rho_{0} \circledast \rho_{1}=\rho_{1}, \rho_{0} \circledast \rho_{2}=\rho_{2}, \rho_{0} \circledast \rho_{3}=\rho_{3}, \rho_{0} \circledast \rho_{4}=\rho_{4}, \rho_{0} \circledast \rho_{5}=\rho_{5} ;$

$\rho_{1} \circledast \rho_{0}=\rho_{1}, \rho_{1} \circledast \rho_{1}=\rho_{1}, \rho_{1} \circledast \rho_{2}=\rho_{1}, \rho_{1} \circledast \rho_{3}=\rho_{3}, \rho_{1} \circledast \rho_{4}=\rho_{4}, \rho_{1} \circledast \rho_{5}=\rho_{5} ;$

$\rho_{2} \circledast \rho_{0}=\rho_{2}, \rho_{2} \circledast \rho_{1}=\rho_{1}, \rho_{2} \circledast \rho_{2}=\rho_{2}, \rho_{2} \circledast \rho_{3}=\rho_{3}, \rho_{2} \circledast \rho_{4}=\rho_{4}, \rho_{2} \circledast \rho_{5}=\rho_{5} ;$

$\rho_{3} \circledast \rho_{0}=\rho_{3}, \rho_{3} \circledast \rho_{1}=\rho_{3}, \rho_{3} \circledast \rho_{2}=\rho_{3}, \rho_{3} \circledast \rho_{3}=\rho_{1}, \rho_{3} \circledast \rho_{4}=\rho_{5}, \rho_{3} \circledast \rho_{5}=\rho_{4} ;$

$\rho_{4} \circledast \rho_{0}=\rho_{4}, \rho_{4} \circledast \rho_{1}=\rho_{4}, \rho_{4} \circledast \rho_{2}=\rho_{4}, \rho_{4} \circledast \rho_{3}=\rho_{5}, \rho_{4} \circledast \rho_{4}=\rho_{3}, \rho_{4} \circledast \rho_{5}=\rho_{1} ;$

$\rho_{5} \circledast \rho_{0}=\rho_{5}, \rho_{5} \circledast \rho_{1}=\rho_{5}, \rho_{5} \circledast \rho_{2}=\rho_{5}, \rho_{5} \circledast \rho_{3}=\rho_{4}, \rho_{5} \circledast \rho_{4}=\rho_{1}, \rho_{5} \circledast \rho_{5}=\rho_{3}$.

Then $M(X)=\left\{\rho_{0}, \rho_{1}, \rho_{2}, \rho_{3}, \rho_{4}, \rho_{5}\right\}$, and $(M(X), \circledast)$ is a monoid, and the operation $\circledast$ on

\begin{tabular}{|c|c|c|c|c|c|c|}
\hline$\circledast$ & $\rho_{0}$ & $\rho_{1}$ & $\rho_{2}$ & $\rho_{3}$ & $\rho_{4}$ & $\rho_{5}$ \\
\hline$\rho_{0}$ & $\rho_{0}$ & $\rho_{1}$ & $\rho_{2}$ & $\rho_{3}$ & $\rho_{4}$ & $\rho_{5}$ \\
\hline$\rho_{1}$ & $\rho_{1}$ & $\rho_{1}$ & $\rho_{1}$ & $\rho_{3}$ & $\rho_{4}$ & $\rho_{5}$ \\
\hline$\rho_{2}$ & $\rho_{2}$ & $\rho_{1}$ & $\rho_{2}$ & $\rho_{3}$ & $\rho_{4}$ & $\rho_{5}$ \\
\hline$\rho_{3}$ & $\rho_{3}$ & $\rho_{3}$ & $\rho_{3}$ & $\rho_{1}$ & $\rho_{5}$ & $\rho_{4}$ \\
\hline$\rho_{4}$ & $\rho_{4}$ & $\rho_{4}$ & $\rho_{4}$ & $\rho_{5}$ & $\rho_{3}$ & $\rho_{1}$ \\
\hline$\rho_{5}$ & $\rho_{5}$ & $\rho_{5}$ & $\rho_{5}$ & $\rho_{4}$ & $\rho_{1}$ & $\rho_{3}$ \\
\hline
\end{tabular}
it is shown in Table 2. Thus $(M(X), \circledast)$ is commutative monoid.

Table 2. The adjoint semigroup of BCI-algebra.

Theorem 6. Assume that $\langle X, *, 0\rangle$ is a generalized quasi-left alter BCI-algebra. Hence, $M(X)$ is a commutative Clifford semigroup. 
Proof. Step 1:

Let $B(X)$ be a $B C K$-part of $X$ and $L(X)=X-B(X)$ be $B C K$-remainder of $X . \forall x \in X$,

$$
\rho_{0} \circledast \rho_{0}(x)=(x * 0) * 0=x * 0=x=\rho_{0}(x) .
$$

Then, according to Definition 6, Proposition 2 and Theorem 4, $\forall x, y \in X-\{0\}$, Case 1: $\forall x, y \in B(X), \forall a \in X, a \neq x$ and $a \neq y$. That is,

$$
\begin{gathered}
\rho_{x} \circledast \rho_{y}(a)=(a * y) * x=a * x=a=\rho_{y}(a)=\rho_{x}(a) . \\
\rho_{x} \circledast \rho_{y}(y)=(y * y) * x=0 * x=0=\rho_{y}(y) . \\
\rho_{x} \circledast \rho_{y}(x)=(x * y) * x=x * x=0=\rho_{x}(x) .
\end{gathered}
$$

Therefore, $\forall x, y \in B(X)$ and $x \neq y$, there is $\rho_{x} \circledast \rho_{y} \in M(X)$.

Then:

(1) $\left(\rho_{x} \circledast \rho_{y}\right) \circledast \rho_{y}(a)=((a * y) * y) * x=(a * y) * x=a * x=(a * y) * x=\rho_{x} \circledast \rho_{y}(a)$; $\left(\rho_{x} \circledast \rho_{y}\right) \circledast \rho_{y}(y)=((y * y) * y) * x=(0 * y) * x=0 * x=(y * y) * x=\rho_{x} \circledast \rho_{y}(y)$; $\left(\rho_{x} \circledast \rho_{y}\right) \circledast \rho_{y}(x)=((x * y) * y) * x=(x * y) * x=x * x=(x * y) * x=\rho_{x} \circledast \rho_{y}(x)$.

Therefore, for any $x, y \in B(X)$, there is $\left(\rho_{x} \circledast \rho_{y}\right) \circledast \rho_{y}=\rho_{x} \circledast \rho_{y}$.

(2) $\left(\rho_{x} \circledast \rho_{y}\right) \circledast \rho_{x}(a)=((a * x) * y) * x=(a * y) * x=a * x=(a * y) * x=\rho_{x} \circledast \rho_{y}(a)$; $\left(\rho_{x} \circledast \rho_{y}\right) \circledast \rho_{x}(y)=((y * x) * y) * x=(y * y) * x=0 * x(y * y) * x=\rho_{x} \circledast \rho_{y}(y)$;

$\left(\rho_{x} \circledast \rho_{y}\right) \circledast \rho_{x}(x)=((x * x) * y) * x=(0 * y) * x=0 * x=0=x * x=(x * y) * x=$ $\rho_{x} \circledast \rho_{y}(x)$.

Therefore, for any $x, y \in B(X)$, there is $\left(\rho_{x} \circledast \rho_{y}\right) \circledast \rho_{x}=\rho_{x} \circledast \rho_{y}$.

(3) $\left(\rho_{x} \circledast \rho_{y}\right) \circledast\left(\rho_{x} \circledast \rho_{y}\right)(a)=(((a * y) * x) * y) * x=((a * x) * y) * x=a * x=$ $(a * y) * x=\rho_{x} \circledast \rho_{y}(a)$;

$\left(\rho_{x} \circledast \rho_{y}\right) \circledast\left(\rho_{x} \circledast \rho_{y}\right)(y)=(((y * y) * x) * y) * x=((0 * x) * y) * x=0 * x=(y * y) *$ $x=\rho_{x} \circledast \rho_{y}(y) ;$

$\left(\rho_{x} \circledast \rho_{y}\right) \circledast\left(\rho_{x} \circledast \rho_{y}\right)(x)=(((x * y) * x) * y) * x=((x * x) * y) * x=0 * x=0=$ $0 * y=(x * x) * y=(x * y) * x=\rho_{x} \circledast \rho_{y}(x)$.

So, for any $x, y \in B(X)$, there is $\left(\rho_{x} \circledast \rho_{y}\right) \circledast\left(\rho_{x} \circledast \rho_{y}\right)=\rho_{x} \circledast \rho_{y}$.

(4) $\forall z \in L(X), b \in L(X)$ and $b \neq z$, and for any $m \in B(X), m \neq x$ and $m \neq y$, there is $\left(\rho_{x} \circledast \rho_{y}\right) \circledast \rho_{z}(m)=((m * z) * y) * x=((0 * z) * y) * x=0 * z=m * z=\rho_{z}(m) ;$

$\left(\rho_{x} \circledast \rho_{y}\right) \circledast \rho_{z}(y)=((y * z) * y) * x=((0 * z) * y) * x=0 * z=y * z=\rho_{z}(y)$;

$\left(\rho_{x} \circledast \rho_{y}\right) \circledast \rho_{z}(x)=((x * z) * y) * x=((0 * z) * y) * x=0 * z=x * z=\rho_{z}(x)$;

$\left(\rho_{x} \circledast \rho_{y}\right) \circledast \rho_{z}(z)=((z * z) * y) * x=(0 * y) * x=0 * x=0=z * z=\rho_{z}(z) ;$

$\left(\rho_{x} \circledast \rho_{y}\right) \circledast \rho_{z}(b)=((b * z) * y) * x=((b * y) * z) * x=(b * z) * x=(b * x) * z=$ $b * z=\rho_{z}(b)$.

That is, for any $x, y \in B(X)$, there is $\left(\rho_{x} \circledast \rho_{y}\right) \circledast \rho_{z}=\rho_{z}$.

Above all, let $|B(X)|=n, a_{i} \in B(X)$ and $i \in[1, n]$. Except for $\rho_{a_{i}} \in M(X)$, there are $\prod_{\in \in[1, n]} \rho_{a_{i}} \in M(X)$. Additionally, for $k, l \in[1, n]$, there is $\left(\rho_{a_{k}} \circledast \ldots \circledast \rho_{a_{l}}\right) \circledast \rho_{a_{k}}=\rho_{a_{k}} \circledast \ldots \circledast \rho_{a_{l}}$, $\left(\rho_{a_{k}} \circledast \ldots \circledast \rho_{a_{l}}\right) \circledast \rho_{a_{l}}=\rho_{a_{k}} \circledast \ldots \circledast \rho_{a_{l}}$. For $j \in[1, n],\left(\prod_{i=1}^{n} \rho_{a_{i}}\right) \circledast \rho_{a_{j}}=\prod_{i=1}^{n} \rho_{a_{i}}$.

Case 2: $\forall x \in B(X), y \in L(X), a \in B(X)$ and $a \neq x, b \in L(X)$ and $b \neq y$. That is:

$$
\begin{gathered}
\rho_{x} \circledast \rho_{y}(a)=(a * y) * x=(0 * y) * x=0 * y=a * y=\rho_{y}(a) . \\
\rho_{x} \circledast \rho_{y}(y)=(y * y) * x=0 * x=0=\rho_{y}(y) . \\
\rho_{x} \circledast \rho_{y}(x)=(x * y) * x=(0 * y) * x=0 * y=x * y=\rho_{y}(x) . \\
\rho_{x} \circledast \rho_{y}(b)=(b * y) * x=b * y=\rho_{y}(b) .
\end{gathered}
$$

Therefore, $\rho_{x} \circledast \rho_{y}=\rho_{y}$. That is:

Case 3: $\forall x \in L(X), y \in B(X)$, For any $a \in B(X)$ and $a \neq y, b \in L(X)$ and $b \neq x$. 


$$
\begin{gathered}
\rho_{x} \circledast \rho_{y}(a)=(a * y) * x=a * x=0 * x=a * x=\rho_{x}(a) . \\
\rho_{x} \circledast \rho_{y}(y)=(y * y) * x=0 * x=y * x=\rho_{x}(y) . \\
\rho_{x} \circledast \rho_{y}(x)=(x * y) * x=x * x=0=\rho_{x}(x) . \\
\rho_{x} \circledast \rho_{y}(b)=(b * y) * x=b * x=\rho_{x}(b) .
\end{gathered}
$$

Therefore, $\rho_{x} \circledast \rho_{y}=\rho_{x}$. That is:

Case 4: $\forall x, y \in L(X)$, For any $a \in B(X), b \in L(X), b \neq x$ and $b \neq y$. Let $z=y *(0 * x)$.

(1) $z \neq 0$, that is, $y \neq 0 * x$,

$$
\begin{aligned}
& \rho_{z}(a)=a * z=0 * z=0 *(y *(0 * x))=(0 * x) * y=(0 * y) * x=(a * y) * x=\rho_{x} \circledast \rho_{y}(a) . \\
& \rho_{z}(y)=y * z=(y * 0) *(y *(0 * x))=(0 * x) * 0=(0 * 0) * x=(y * y) * x=\rho_{x} \circledast \rho_{y}(y) . \\
& \rho_{z}(x)=x * z=(0 *(0 * x)) *(y *(0 * x))=0 * y=(x * x) * y=(x * y) * x=\rho_{x} \circledast \rho_{y}(x) . \\
& \rho_{z}(b)=b * z=(0 *(0 * b)) *(y *(0 * x)) \\
& =(0 *(y *(0 * x)))(0 * b) \\
& =((0 * x) * y) *(0 * b) \\
& =((0 * x) *(0 * b)) * y \\
& =(b * x) * y=(b * y) * x=\rho_{x} \circledast \rho_{y}(b) .
\end{aligned}
$$

Therefore, $\rho_{x} \circledast \rho_{y}=\rho_{y *(0 * x)}$. According to Case 2 and Case $3, \rho_{x} \circledast \rho_{y}$ is a completely regular element.

(2) $z=0$, that is, $y=0 * x$ and $x=0 * y$.

$$
\begin{gathered}
\rho_{x} \circledast \rho_{y}(y)=(y * y) * x=0 * x=y=y * 0=\rho_{0}(y) . \\
\rho_{x} \circledast \rho_{y}(x)=(x * y) * x=(x * x) * y=0 * y=x=x * 0=\rho_{0}(x) . \\
\rho_{x} \circledast \rho_{y}(b)=(b * y) * x=(b * y) *(0 * y)=b * 0=\rho_{0}(b) .
\end{gathered}
$$

However

$$
\rho_{x} \circledast \rho_{y}(a)=(a * y) * x=(0 * y) * x=x * x=0 \neq \rho_{0}(a) .
$$

Therefore, $\rho_{x} * \rho_{y} \in M(X)$.

(i) Let $m \in B(X)$, and $m \neq a$, then:

$\left(\rho_{x} \circledast \rho_{y}\right) \circledast \rho_{m}(a)=((a * m) * y) * x=(a * y) * x=(0 * y) * x=(a * y) * x=$ $\rho_{x} \circledast \rho_{y}(a) ;$

$\left(\rho_{x} \circledast \rho_{y}\right) \circledast \rho_{m}(m)=((m * m) * y) * x=(0 * y) * x=0, \rho_{x} \circledast \rho_{y}(m)=(m * y) * x=$ $(0 * y) * x=0$;

$\left(\rho_{x} \circledast \rho_{y}\right) \circledast \rho_{m}(y)=((y * m) * y) * x=(y * y) * x=\rho_{x} \circledast \rho_{y}(y) ;$

$\left(\rho_{x} \circledast \rho_{y}\right) \circledast \rho_{m}(x)=((x * m) * y) * x=(x * y) * x=\rho_{x} \circledast \rho_{y}(x) ;$

$\left(\rho_{x} \circledast \rho_{y}\right) \circledast \rho_{m}(b)=((b * m) * y) * x=(b * y) * x=\rho_{x} \circledast \rho_{y}(b)$.

Therefore, for $x, y \in L(X)$ and $y=0 * x, m \in B(X)$, there is $\left(\rho_{x} \circledast \rho_{y}\right) \circledast \rho_{m}=\rho_{x} \circledast \rho_{y}$. (ii) Let $m \in L(X)$, and $m \neq x, m \neq y$; then,

$\left(\rho_{x} \circledast \rho_{y}\right) \circledast \rho_{m}(a)=((a * m) * y) * x=((0 * m) * y) * x=((0 * m) *(0 * x)) * x=$ $(x * m) * x=(x * x) * m=0 * m=a * m=\rho_{m}(a)$;

$\left(\rho_{x} \circledast \rho_{y}\right) \circledast \rho_{m}(y)=((y * m) * y) * x=((y * y) * m) * x=(0 * m) * x=(0 * x) * m=$ $y * m=\rho_{m}(y)$;

$\left(\rho_{x} \circledast \rho_{y}\right) \circledast \rho_{m}(x)=((x * m) * y) * x=((x * m) * x) * y=((x * x) * m) * y=(0 *$ $m) * y=(0 * y) * m=x * m=\rho_{m}(x)$;

$\left(\rho_{x} \circledast \rho_{y}\right) \circledast \rho_{m}(m)=((m * m) * y) * x=(0 * y) * x=x * x=0=m * m=\rho_{m}(m) ;$ 
$\left(\rho_{x} \circledast \rho_{y}\right) \circledast \rho_{m}(b)=((b * m) * y) * x=((b * y) * m) * x=((b * y) * x) * m=((b *$ $y) *(0 * y)) * m=(b * 0) * m=b * m=\rho_{m}(b)$.

Therefore, for $x, y \in L(X)$ and $y=0 * x, m \in L(X)$, there is $\left(\rho_{x} \circledast \rho_{y}\right) \circledast \rho_{m}=\rho_{m}$.

(iii) $\left(\rho_{x} \circledast \rho_{y}\right) \circledast \rho_{y}(a)=((a * y) * y) * x=((0 * y) * y) * x=(x * y) * x=(x * x) * y=$ $0 * y=a * y=\rho_{y}(a)$;

$\left(\rho_{x} \circledast \rho_{y}\right) \circledast \rho_{y}(y)=((y * y) * y) * x=(0 * y) * x=x * x=y * y=\rho_{y}(y) ;$

$\left(\rho_{x} \circledast \rho_{y}\right) \circledast \rho_{y}(x)=((x * y) * y) * x=((x * y) * x) * y=((x * x) * y) * y=(0 * y) * y=$ $x * y=\rho_{y}(x)$

$\left(\rho_{x} \circledast \rho_{y}\right) \circledast \rho_{y}(b)=((b * y) * y) * x=((b * y) * x) * y=((b * y) *(0 * y)) * y=$ $(b * 0) * y=b * y=\rho_{y}(b)$;

Therefore, for any $x, y \in L(X)$ and $y=0 * x$, there is $\left(\rho_{x} \circledast \rho_{y}\right) \circledast \rho_{y}=\rho_{y}$.

(iv) $\left(\rho_{x} \circledast \rho_{y}\right) \circledast \rho_{x}(a)=((a * x) * y) * x=((0 * x) * y) * x=(y * y) * x=0 * x=$ $a * x=\rho_{x}(a)$;

$\left(\rho_{x} \circledast \rho_{y}\right) \circledast \rho_{x}(y)=((y * x) * y) * x=((y * y) * x) * x=(0 * x) * x=y * x=\rho_{x}(y) ;$

$\left(\rho_{x} \circledast \rho_{y}\right) \circledast \rho_{x}(x)=((x * x) * y) * x=(0 * y) * x=x * x=\rho_{x}(x)$; $\rho_{x}(b)$

$\left(\rho_{x} \circledast \rho_{y}\right) \circledast \rho_{x}(b)=((b * x) * y) * x=((b * x) *(0 * x)) * x=(b * 0) * x=b * x=$

Therefore, for any $x, y \in L(X)$ and $y=0 * x$, there is $\left(\rho_{x} \circledast \rho_{y}\right) \circledast \rho_{x}=\rho_{x}$.

(v) $\left(\rho_{x} \circledast \rho_{y}\right) \circledast\left(\rho_{x} \circledast \rho_{y}\right)(a)=(((a * y) * x) * y) * x=(((0 * y) *(0 * y)) * y) * x=$ $(a * y) * x=\rho_{x} \circledast \rho_{y}(a)$;

$\left(\rho_{x} \circledast \rho_{y}\right) \circledast\left(\rho_{x} \circledast \rho_{y}\right)(y)=(((y * y) * x) * y) * x=((0 * x) * y) * x=(y * y) * x=$ $\rho_{x} \circledast \rho_{y}(y) ;$

$\left(\rho_{x} \circledast \rho_{y}\right) \circledast\left(\rho_{x} \circledast \rho_{y}\right)(x)=(((x * y) * x) * y) * x=(((x * x) * y) * y) * x=((0 * y) *$ $y) * x=(x * y) * x=\rho_{x} \circledast \rho_{y}(x)$;

$\left(\rho_{x} \circledast \rho_{y}\right) \circledast\left(\rho_{x} \circledast \rho_{y}\right)(b)=(((b * y) * x) * y) * x=((b * y) *(0 * y) * y) * x=((b *$ $0) * y) * x=(b * y) * x=\rho_{x} \circledast \rho_{y}(b)$.

Therefore, for any $x, y \in L(X)$ and $y=0 * x$, there is $\left(\rho_{x} \circledast \rho_{y}\right) \circledast\left(\rho_{x} \circledast \rho_{y}\right)=\rho_{x} \circledast \rho_{y}$. At the same time, $\rho_{x} \circledast \rho_{y}$ is idempotent.

In sum, all elements of $M(X)$ are aligned.

Step 2:

Case 1: According to Case1 in Step1, let $|B(X)|=n, x, a_{i} \in B(X)$ and $i \in[1, n]$, $\forall a \in X$. Then, $\rho_{x} \circledast \rho_{x}(a)=(a * x) * x=a * x=\rho_{x}, \rho_{x}$ is idempotent and a completely regular element. $\left(\prod_{i \in[1, n]} \rho_{a_{i}}\right) \circledast\left(\prod_{i \in[1, n]} \rho_{a_{i}}\right)=\prod_{i \in[1, n]} \rho_{a_{i}}$. That is, $\prod_{i \in[1, n]} \rho_{a_{i}}$ is idempotent and a completely regular element.

Case 2: $\forall x \in B(X), y \in L(X), b \in L(X)$ and $b \neq y$. That is, there exists $z=0 * y$ and $y=0 * z$. Then,

(1)

$$
\begin{gathered}
\rho_{y} \circledast \rho_{z} \circledast \rho_{y}(a)=((a * y) * z) * y=((0 * y) *(0 * y)) * y=0 * y=a * y=\rho_{y}(a) . \\
\rho_{z} \circledast \rho_{y} \circledast \rho_{z}(a)=((a * z) * y) * z=((0 * z) *(0 * z)) * z=0 * z=a * z=\rho_{z}(a) . \\
\rho_{z} \circledast \rho_{y}(a)=(a * y) * z=(a * z) * y=\rho_{y} \circledast \rho_{z}(a) .
\end{gathered}
$$

$$
\begin{gathered}
\rho_{y} \circledast \rho_{z} \circledast \rho_{y}(y)=((y * y) * z) * y=(0 * z) * y=y * y=0=y * y=\rho_{y}(y) . \\
\rho_{z} \circledast \rho_{y} \circledast \rho_{z}(y)=((y * z) * y) * z=((y * y) * z) * z=(0 * z) * z=y * z=\rho_{z}(y) . \\
\rho_{z} \circledast \rho_{y}(y)=(y * y) * z=(y * z) * y=\rho_{y} \circledast \rho_{z}(y) .
\end{gathered}
$$

$$
\begin{gathered}
\rho_{y} \circledast \rho_{z} \circledast \rho_{y}(z)=((z * y) * z) * y=((z * z) * y) * y=(0 * y) * y=z * y=\rho_{y}(z) . \\
\rho_{z} \circledast \rho_{y} \circledast \rho_{z}(z)=((z * z) * y) * z=(0 * y) * z=z * z=\rho_{z}(z) .
\end{gathered}
$$




$$
\rho_{z} \circledast \rho_{y}(z)=(z * y) * z=(z * z) * y=\rho_{y} \circledast \rho_{z}(z)
$$

(4)

$$
\begin{gathered}
\rho_{y} \circledast \rho_{z} \circledast \rho_{y}(b)=((b * y) * z) * y=((b * y) *(0 * y)) * y=(b * 0) * y=b * y=\rho_{y}(b) . \\
\rho_{z} \circledast \rho_{y} \circledast \rho_{z}(b)=((b * z) * y) * z=((b * z) *(0 * z)) * z=(b * 0) * z=b * z=\rho_{z}(b) . \\
\rho_{z} \circledast \rho_{y}(b)=(b * y) * z=(b * z) * y=\rho_{y} \circledast \rho_{z}(b) .
\end{gathered}
$$

Above all, $\rho_{y}$ is a completely regular element.

Case 3:

According to Case 4 in Step 1, $\forall x, y \in L(X)$, and $y \neq 0 * x, \rho_{x} \circledast \rho_{y}=\rho_{y *(0 * x)}$. According to Case $2, \rho_{x} \circledast \rho_{y}$ is a completely regular element. Additionally, $\left(\rho_{x} \circledast \rho_{0 * x}\right) \circledast$ $\left(\rho_{x} \circledast \rho_{0 * x}\right)=\rho_{x} \circledast \rho_{0 * x}$. At the same time, $\rho_{x} \circledast \rho_{0 * x}$ is idempotent. Additionally, $\left(\rho_{y} \circledast\right.$ $\left.\rho_{0 * y}\right) \circledast\left(\rho_{y} \circledast \rho_{0 * y}\right)=\rho_{y} \circledast \rho_{0 * y}$. At the same time, $\rho_{y} \circledast \rho_{0 * y}$ is idempotent.

Therefore, $M(X)$ is a completely regular semigroup. In addition, $M(X)$ is a commutative Clifford semigroup because of commutativity.

Example 2. Assume that $X=\{0,1,2,3,4,5\}$. The operation on $X$ is shown in Table 3 .

Table 3. Generalized quasi-left alter BCI-algebra.

\begin{tabular}{lllllll}
\hline$*$ & $\mathbf{0}$ & $\mathbf{1}$ & $\mathbf{2}$ & $\mathbf{3}$ & $\mathbf{4}$ & $\mathbf{5}$ \\
\hline 0 & 0 & 0 & 0 & 3 & 5 & 4 \\
1 & 1 & 0 & 1 & 3 & 5 & 4 \\
2 & 2 & 2 & 0 & 3 & 5 & 4 \\
3 & 3 & 3 & 3 & 0 & 4 & 5 \\
4 & 4 & 4 & 4 & 5 & 0 & 3 \\
5 & 5 & 5 & 5 & 4 & 3 & 0 \\
\hline
\end{tabular}

Then, $<X, *, 0>$ is a generalized quasi-left alter BCI-algebra and $M(X)=\left\{\rho_{0}, \rho_{1}, \rho_{2}, \rho_{3}, \rho_{4}\right.$, $\left.\rho_{5}, \rho_{3}^{2}\right\}$, where $\rho_{3}^{2}=\rho_{3} * \rho_{3}$.

We can verify the following:

$\rho_{0} \circledast \rho_{0}=\rho_{0}, \rho_{0} \circledast \rho_{1}=\rho_{1}, \rho_{0} \circledast \rho_{2}=\rho_{2}, \rho_{0} \circledast \rho_{3}=\rho_{3}, \rho_{0} \circledast \rho_{4}=\rho_{4}, \rho_{0} \circledast \rho_{5}=$ $\rho_{5}, \rho_{0} \circledast \rho_{3}^{2}=\rho_{3}^{2}$;

$\rho_{1} \circledast \rho_{0}=\rho_{1}, \rho_{1} \circledast \rho_{1}=\rho_{1}, \rho_{1} \circledast \rho_{2}=\rho_{3}^{2}, \rho_{1} \circledast \rho_{3}=\rho_{3}, \rho_{1} \circledast \rho_{4}=\rho_{4}, \rho_{1} \circledast \rho_{5}=$ $\rho_{5}, \rho_{1} \circledast \rho_{3}^{2}=\rho_{3}^{2}$;

$\rho_{2} \circledast \rho_{0}=\rho_{2}, \rho_{2} \circledast \rho_{1}=\rho_{3}^{2}, \rho_{2} \circledast \rho_{2}=\rho_{2}, \rho_{2} \circledast \rho_{3}=\rho_{3}, \rho_{2} \circledast \rho_{4}=\rho_{4}, \rho_{2} \circledast \rho_{5}=$ $\rho_{5}, \rho_{2} \circledast \rho_{3}^{2}=\rho_{3}^{2}$;

$\rho_{3} \circledast \rho_{0}=\rho_{3}, \rho_{3} \circledast \rho_{1}=\rho_{3}, \rho_{3} \circledast \rho_{2}=\rho_{3}, \rho_{3} \circledast \rho_{3}=\rho_{3}^{2}, \rho_{2} \circledast \rho_{4}=\rho_{5}, \rho_{2} \circledast \rho_{5}=$ $\rho_{4}, \rho_{3} \circledast \rho_{3}^{2}=\rho_{3}$;

$\rho_{4} \circledast \rho_{0}=\rho_{4}, \rho_{4} \circledast \rho_{1}=\rho_{4}, \rho_{4} \circledast \rho_{2}=\rho_{4}, \rho_{4} \circledast \rho_{3}=\rho_{5}, \rho_{4} \circledast \rho_{4}=\rho_{3}, \rho_{4} \circledast \rho_{5}=$ $\rho_{3}^{2}, \rho_{4} \circledast \rho_{3}^{2}=\rho_{4}$;

$\rho_{5} \circledast \rho_{0}=\rho_{5}, \rho_{5} \circledast \rho_{1}=\rho_{5}, \rho_{5} \circledast \rho_{2}=\rho_{5}, \rho_{5} \circledast \rho_{3}=\rho_{4}, \rho_{5} \circledast \rho_{4}=\rho_{3}^{2}, \rho_{5} \circledast \rho_{5}=$ $\rho_{3}, \rho_{5} \circledast \rho_{3}^{2}=\rho_{5}$;

$\rho_{3}^{2} \circledast \rho_{0}=\rho_{3}^{2}, \rho_{3}^{2} \circledast \rho_{1}=\rho_{3}^{2}, \rho_{3}^{2} \circledast \rho_{2}=\rho_{3}^{2}, \rho_{3}^{2} \circledast \rho_{3}=\rho_{3}, \rho_{3}^{2} \circledast \rho_{4}=\rho_{4}, \rho_{3}^{2} \circledast \rho_{5}=$ $\rho_{5}, \rho_{3}^{2} \circledast \rho_{3}^{2}=\rho_{3}^{2}$.

Then $M(X)$ is a completely regular semigroup, and the operation $\circledast$ on it is shown in Table 4 . 
Table 4. The adjoint semigroup of generalized quasi left alter BCI-algebra.

\begin{tabular}{cccccccc}
\hline$\circledast$ & $\rho_{\mathbf{0}}$ & $\rho_{\mathbf{1}}$ & $\rho_{\mathbf{2}}$ & $\rho_{\mathbf{3}}$ & $\boldsymbol{\rho}_{\mathbf{4}}$ & $\boldsymbol{\rho}_{\mathbf{5}}$ & $\boldsymbol{\rho}_{\mathbf{3}}^{\mathbf{2}}$ \\
\hline$\rho_{0}$ & $\rho_{0}$ & $\rho_{1}$ & $\rho_{2}$ & $\rho_{3}$ & $\rho_{4}$ & $\rho_{5}$ & $\rho_{3}^{2}$ \\
$\rho_{1}$ & $\rho_{1}$ & $\rho_{1}$ & $\rho_{3}$ & $\rho_{3}$ & $\rho_{4}$ & $\rho_{5}$ & $\rho_{3}^{2}$ \\
$\rho_{2}$ & $\rho_{2}$ & $\rho_{3}$ & $\rho_{2}$ & $\rho_{3}$ & $\rho_{4}$ & $\rho_{5}$ & $\rho_{3}^{2}$ \\
$\rho_{3}$ & $\rho_{3}$ & $\rho_{3}$ & $\rho_{3}$ & $\rho_{3}^{2}$ & $\rho_{5}$ & $\rho_{4}$ & $\rho_{3}$ \\
$\rho_{4}$ & $\rho_{4}$ & $\rho_{4}$ & $\rho_{4}$ & $\rho_{5}$ & $\rho_{3}$ & $\rho_{3}^{2}$ & $\rho_{4}$ \\
$\rho_{5}$ & $\rho_{5}$ & $\rho_{5}$ & $\rho_{5}$ & $\rho_{4}$ & $\rho_{3}^{2}$ & $\rho_{3}$ & $\rho_{5}$ \\
$\rho_{3}^{2}$ & $\rho_{3}^{2}$ & $\rho_{3}^{2}$ & $\rho_{3}^{2}$ & $\rho_{3}$ & $\rho_{4}$ & $\rho_{5}$ & $\rho_{3}^{2}$ \\
\hline
\end{tabular}

In the following, we introduce QM- $B C I$ algebra and discuss the relationship between QM-BCI algebra and a generalized quasi-left alter $B C I$-algebra.

Definition 15. Assume that $(X, \leq)$ is a partial order containing a constant $0, x \in X . x$ is said to be a quasi-minimal element, if $\forall a \in X, a \leq x$ implies $x=a$ or $a=0$.

Definition 16. A BCI-algebra $(X, \leq, *, 0)$ is called a $Q M-B C I$ algebra, if all elements of $X$ are quasi-minimal elements.

Theorem 7. Assume that $(X, \leq, *, 0)$ is a BCI-algebra. $X$ is a QM-BCI algebra if it meets: $\forall x, y \in X-\{0\}$

$$
x \leq y \text { implies } x=y
$$

Proof. $(\Rightarrow) \forall x, y \in X-\{0\}$, assume that $x \leq y$, according to Definition $15, y=x$ or $x=0$. However, $x \neq 0$. So $x=y$.

$(\Leftarrow)$ Assume that $x, y \in X, x \leq y$. If $y=0$, then $x \leq y=0$, we can get $x=y=0$. If $x \neq 0, y \neq 0$, there is $x=y$ by condition. Therefore, $y$ is a quasi-minimal element of $X$. Thus, $X$ is a QM-BCI algebra.

Theorem 8. Assume that $(X, \leq, *, 0)$ is a BCI-algebra, $B(X)$ is a BCK-part of $X, A G(X)$ is a generalized associative part of $X$. Then, the below conditions are equivalent:

(1) $X$ is a $Q M-B C I$ algebra;

(2) $B(X)$ is quasi-alter $B C K$-algebra and $A G(X)=(X-B(X)) \cup\{0\}$;

(3) $X$ is a generalized quasi-left alter BCI-algebra.

Proof. (1) $\Rightarrow(2)$ Assume that $X$ is a QM-BCI algebra. Then, for any $x, y \in B(X)$, if $x=y$, $x * y=0$. If $x \neq y$, it could be divided into the following three cases:

Case 1: $x=0, y \neq 0, x *(x * y)=0 *(0 * y)=0 * 0=0$, that is, $x \leq x * y$; $(x * y) * x=(0 * y) * 0=0 * 0=0$, that is, $x * y \leq x$. According to Definition $4, x=x * y$.

Case 2: $x \neq 0, y=0, x *(x * y)=x *(x * 0)=x * x=0$, that is, $x \leq x * y$; $(x * y) * x=(x * 0) * x=x * x=0$, that is, $x * y \leq x$. According to Definition $4, x=x * y$.

Case 3: $x, y \neq 0,(x * y) * x=(x * x) * y=0 * y=0$, that is, $x \leq x * y$. Because $x * y \neq 0$ and $x \neq 0$, according to Theorem $7, x * y=x$.

According to Definition 6, $B(X)$ is a quasi-alter $B C K$-algebra. If $x \in X-B(X)$, then $0 * x \neq 0$ and $0 *(0 * x) \neq 0$. As $(0 *(0 * x)) * x=(0 * x) *(0 * x)=0$, that is, $0 *(0 * x) \leq x$. According to Theorem $7,0 *(0 * x)=x$. Thus, $(X-B(X)) \cup\{0\} \subseteq A G(X)$. On the other hand, $A G(X) \subseteq(X-B(X)) \cup\{0\}$; then, $A G(X)=(X-B(X)) \cup\{0\}$.

(2) $\Rightarrow(3) \forall x, y \in X, x \neq y$,

Case 1: $\forall x, y \in B(X), x *(x * y)=x * x=0,0 *(0 * y)=0 * 0=0$. Then, $x *(x * y)=$ $0 *(0 * y)$.

Case 2: $\forall x, y \in A G(X)$, assume that $x \leq y$, that is, $x * y=0$. Then,

$$
0 *(y * x)=(x * x) *(y * x) \leq x * y=0 .
$$


Therefore, $0 *(y * x)=0$. Then, $y * x=0 *(0 *(y * x))=0 * 0=0$, and $y * x=0$. That is, $y \leq x$. So $x=y$. According to Definition 4 , we can see that $x *(x * y) \leq y$; that is, $x *(x * y)=y$. Additionally, $0 *(0 * y)=y$. So $x *(x * y)=0 *(0 * y)$.

Case 3: $\forall x \in B(X), \forall y \in A G(X)$, according to Theorem $4, x * y=0 * y$; then, $x *(x *$ $y)=0 *(0 * y)$.

Case 4: $\forall x \in A G(X), \forall y \in B(X)$, according to Definition $6, x * y=x$; then, $x *(x *$ $y)=x * x=0$, and $0 *(0 * y)=0 * 0=0$. So $x *(x * y)=0 *(0 * y)$.

Above all, $X$ is a generalized quasi-left alter BCI-algebra. Then,

$(3) \Rightarrow(1)$ If $X$ is a generalized quasi-left alter $B C I$-algebra, assume that $x \leq y$ and $x \neq y$.

$$
x=x * 0=x *(x * y)=0 *(0 * y) .
$$

$\forall y \in X$

Case 1: If $y \in B(X)$, then $x=0 *(0 * y)=0 * 0=0$;

Case 2: If $y \in A G(X)$, then $x=0 *(0 * y)=y$. As $x \neq y$, then $x=0$. Thus, for any $y \in X, y$ is a quasi-minimal element of $X$.

In the following, we propose the adjoint semigroup of hyper $B C I$-algebra and replace the singleton set $\{x\}$ with $x$. Additionally, the concepts of generalized quasi-left alter-hyper $B C I$-algebra and QM-hyper BCI are shown.

Let $(H, \circ)$ be a hyper $B C I$-algebra. $\forall a, x \in H$, denote a map:

$$
\rho_{a}: H \rightarrow P^{*}(H) ; x \mapsto x \circ a .
$$

where $P^{*}(H)$ represents the non-empty subset of $H$.

$\forall a, b \in H$, denote $\rho_{a} \circledast \rho_{b}: H \rightarrow P^{*}(H)$ as follows: $\forall x \in H$,

$$
x \mapsto\left(\rho_{a} \circledast \rho_{b}\right)(x)=\bigcup_{\forall y \in \rho_{b}(x)} \rho_{a}(y) .
$$

where $\circledast$ represents the composition operation.

Theorem 9. Assume that $(H, \circ)$ is a hyper BCI-algebra. Denote $M(H)$ as a set of finite products $\rho_{a_{1}} \circledast \ldots \circledast \rho_{a_{s}}\left(a_{1}, \ldots, a_{s} \in H\right)$, where $\circledast$ represents the composition operation of mappings. Then, $M(H)$ is a commutative semigroup.

Proof. $\forall x \in H, a, b, c \in H$, for any $s \in\left(\left(\rho_{a} \circledast \rho_{b}\right) \circledast \rho_{c}\right)(x), \exists y \in \rho_{c}(x)$ s.t. $s \in\left(\rho_{a} \circledast \rho_{b}\right)(y)$. Then $\exists u \in \rho_{b}(y)$ s.t. $u \in \rho_{b}\left(\rho_{c}(x)\right)=\rho_{b} \circledast \rho_{\mathcal{c}}(x)$ and $s \in \rho_{a}(u)$. Then $s \in\left(\rho_{a} \circledast\left(\rho_{b} \circledast \rho_{\mathcal{c}}\right)\right)(x)$ and $\left(\left(\rho_{a} \circledast \rho_{b}\right) \circledast \rho_{c}\right)(x) \subseteq\left(\rho_{a} \circledast\left(\rho_{b} \circledast \rho_{c}\right)\right)(x)$.

For any $t \in\left(\rho_{a} \circledast\left(\rho_{b} \circledast \rho_{c}\right)\right)(x)$, there exists $m \in \rho_{b} \circledast \rho_{c}(x)$ such that $t \in \rho_{a}(m)$. Then $\exists n \in \rho_{c}(x)$ s.t. $m \in \rho_{b}(n)$ and $t \in \rho_{a}\left(\rho_{b}(n)\right)=\rho_{a} \circledast \rho_{b}(n)$. Then $t \in\left(\left(\rho_{a} \circledast \rho_{b}\right) \circledast \rho_{c}\right)(x)$ and $\left(\rho_{a} \circledast\left(\rho_{b} \circledast \rho_{c}\right)\right)(x) \subseteq\left(\left(\rho_{a} \circledast \rho_{b}\right) \circledast \rho_{\mathcal{c}}\right)(x)$.

Therefore, $\left(\rho_{a} \circledast\left(\rho_{b} \circledast \rho_{c}\right)\right)(x)=\left(\left(\rho_{a} \circledast \rho_{b}\right) \circledast \rho_{c}\right)(x)$. Then, $M(H)$ satisfies the associative law.

For any $m, n \in H, x \in H . \rho_{m} \circledast \rho_{n}(x)=(x \circ n) \circ m=(x \circ m) \circ n=\rho_{n} \circledast \rho_{m}(x)$. Therefore, $M(H)$ satisfies the commutative law. Thus, $M(H)$ is a commutative semigroup.

Example 3. Assume that $H=\{0,1,2,3,4\}$. Define the operation on $H$ in Table 5,

Table 5. Hyper BCI-algebra.

\begin{tabular}{cccccc}
\hline$\circ$ & $\mathbf{0}$ & $\mathbf{1}$ & $\mathbf{2}$ & $\mathbf{3}$ & $\mathbf{4}$ \\
\hline 0 & 0 & 0 & 0 & 4 & 3 \\
1 & $\{1,2\}$ & $\{0,1,2\}$ & $\{0,1,2\}$ & 4 & 3 \\
2 & 2 & $\{1,2\}$ & $\{0,1,2\}$ & 4 & 3 \\
3 & 3 & 3 & 3 & 0 & 4 \\
4 & 4 & 4 & 4 & 3 & 0 \\
\hline
\end{tabular}


Clearly, $(H, \circ)$ is a hyper BCI-algebra.

That is,

$\rho_{0}: H \rightarrow H: x \mapsto x \circ 0$. So $\rho_{0}=\{0,\{1,2\}, 2,3,4\}$;

$\rho_{1}: H \rightarrow H: x \mapsto x \circ 1$. So $\rho_{1}=\{0,\{0,1,2\},\{1,2\}, 3,4\}$;

$\rho_{2}: H \rightarrow H: x \mapsto x \circ 2$. So $\rho_{2}=\{0,\{0,1,2\},\{0,1,2\}, 3,4\}$;

$\rho_{3}: H \rightarrow H: x \mapsto x \circ 3$. So $\rho_{3}=\{4,4,4,0,3\}$;

$\rho_{4}: H \rightarrow H: x \mapsto x \circ 4$. So $\rho_{4}=\{3,3,3,4,0\}$.

Denote $\rho_{34}=\rho_{3} \circledast \rho_{4}=\{0,0,0,3,4\}$.

We can verify the following:

$\rho_{0} \circledast \rho_{0}=\rho_{0}, \rho_{0} \circledast \rho_{1}=\rho_{1}, \rho_{0} \circledast \rho_{2}=\rho_{2}, \rho_{0} \circledast \rho_{3}=\rho_{3}, \rho_{0} \circledast \rho_{4}=\rho_{4}, \rho_{0} \circledast \rho_{34}=\rho_{34} ;$

$\rho_{1} \circledast \rho_{0}=\rho_{1}, \rho_{1} \circledast \rho_{1}=\rho_{2}, \rho_{1} \circledast \rho_{2}=\rho_{2}, \rho_{1} \circledast \rho_{3}=\rho_{3}, \rho_{1} \circledast \rho_{4}=\rho_{4}, \rho_{1} \circledast \rho_{34}=\rho_{34} ;$

$\rho_{2} \circledast \rho_{0}=\rho_{2}, \rho_{2} \circledast \rho_{1}=\rho_{2}, \rho_{2} \circledast \rho_{2}=\rho_{2}, \rho_{2} \circledast \rho_{3}=\rho_{3}, \rho_{2} \circledast \rho_{4}=\rho_{4}, \rho_{2} \circledast \rho_{34}=\rho_{34} ;$

$\rho_{3} \circledast \rho_{0}=\rho_{3}, \rho_{3} \circledast \rho_{1}=\rho_{3}, \rho_{3} \circledast \rho_{2}=\rho_{3}, \rho_{3} \circledast \rho_{3}=\rho_{4}, \rho_{3} \circledast \rho_{4}=\rho_{34}, \rho_{3} \circledast \rho_{34}=\rho_{3} ;$

$\rho_{4} \circledast \rho_{0}=\rho_{4}, \rho_{4} \circledast \rho_{1}=\rho_{4}, \rho_{4} \circledast \rho_{2}=\rho_{4}, \rho_{4} \circledast \rho_{3}=\rho_{34}, \rho_{4} \circledast \rho_{4}=\rho_{3}, \rho_{4} \circledast \rho_{34}=\rho_{4} ;$

$\rho_{34} \circledast \rho_{0}=\rho_{34}, \rho_{34} \circledast \rho_{1}=\rho_{34}, \rho_{34} \circledast \rho_{2}=\rho_{34}, \rho_{34} \circledast \rho_{3}=\rho_{3}, \rho_{34} \circledast \rho_{4}=\rho_{4}, \rho_{34} \circledast \rho_{34}=$ $\rho_{34}$.

Then $M(H)=\left\{\rho_{0}, \rho_{1}, \rho_{2}, \rho_{3}, \rho_{4}, \rho_{34}\right\},(M(H), \circledast)$ is a commutative semigroup, and the operation on it is shown in Table 6.

Table 6. The adjoint semigroup of hyper BCI-algebra.

\begin{tabular}{cccccccc}
\hline$\circledast$ & $\rho_{\mathbf{0}}$ & $\rho_{\mathbf{1}}$ & $\rho_{\mathbf{2}}$ & $\rho_{\mathbf{3}}$ & & $\boldsymbol{\rho}_{\mathbf{4}}$ & $\rho_{\mathbf{3 4}}$ \\
\hline$\rho_{0}$ & $\rho_{0}$ & $\rho_{1}$ & $\rho_{2}$ & $\rho_{3}$ & & $\rho_{4}$ & $\rho_{34}$ \\
$\rho_{1}$ & $\rho_{1}$ & $\rho_{2}$ & $\rho_{2}$ & $\rho_{3}$ & $\rho_{4}$ & $\rho_{34}$ \\
$\rho_{2}$ & $\rho_{2}$ & $\rho_{2}$ & $\rho_{2}$ & $\rho_{3}$ & $\rho_{4}$ & $\rho_{34}$ \\
$\rho_{3}$ & $\rho_{3}$ & $\rho_{3}$ & $\rho_{3}$ & $\rho_{4}$ & $\rho_{34}$ & $\rho_{3}$ \\
$\rho_{4}$ & $\rho_{4}$ & $\rho_{4}$ & $\rho_{4}$ & $\rho_{34}$ & $\rho_{3}$ & $\rho_{4}$ \\
$\rho_{34}$ & $\rho_{34}$ & $\rho_{34}$ & $\rho_{34}$ & $\rho_{3}$ & $\rho_{4}$ & $\rho_{34}$ \\
\hline
\end{tabular}

Definition 17. In hyper BCI-algebra $(H, \circ)$, if $\forall x, y \in H$, and $x \neq y$,

$$
x \circ(x \circ y)=0 \circ(0 \circ y) .
$$

Then, it is a generalized quasi-left alter-hyper BCI-algebra.

Theorem 10. Assume that $(H, 0)$ is a generalized quasi-left alter-hyper BCI-algebra. Hence, $(H, 0)$ is BCI-algebra.

Proof. Assume that $(H, \circ)$ is a generalized quasi-left alter-hyper $B C I$-algebra. $\forall x \in H$ and $x \neq 0,0=0 \circ 0 \in(x \circ x) \circ 0=(x \circ 0) \circ x$. Then, $\exists p \in x \circ 0$, such that $0 \in p \circ x$; that is, $p \ll x$. According to Definition 14, $x \circ p \subseteq x \circ(x \circ 0)=0 \circ(0 \circ 0)=0$. That is to say, $x \ll p$. According to Definition 14, $x=p$. Therefore, $x \in x \circ 0$. Then, $x \circ x \subseteq x \circ(x \circ 0)=$ $0 \circ(0 \circ 0)=0$.

$\forall x, y \in H$, assume that $|x \circ y|>1$. Let $m, n \in x \circ y$ and $m \neq n$. Then, $m \circ n \subseteq$ $(x \circ y) \circ(x \circ y) \ll x \circ x=0, n \circ m \subseteq(x \circ y) \circ(x \circ y) \ll x \circ x=0$. According to Definition $13, m \circ n=0, n \circ m=0$, that is, $m \ll n, n \ll m$. Additionally, $m=n$. Therefore, $|x \circ y|=1$.

As $x \in x \circ 0, x=x \circ 0$. Additionally, $H$ is BCI-algebra.

Definition 18. Let $(H, \ll)$ be a partial order containing 0 in hyper structure. $x$ is said to be a quasi-minimal element in $H$. If for any element $a$ in $H, a \ll x \Rightarrow x=a$ or $a=0$.

Definition 19. A hyper BCI-algebra $(H, \ll, \circ, 0)$ is called a QM-hyper BCI algebra if all elements of $H$ are quasi-minimal elements. 
Theorem 11. A hyper BCI-algebra $(H, \ll, \circ, 0)$ is a QM-hyper BCI algebra if it meets: $\forall x, y \in$ $H-\{0\}$,

$$
x \ll y \text { implies } x=y \text {. }
$$

Proof. $(\Rightarrow) \forall x, y \in H-\{0\}$, assume that $x \ll y$, according to Definition $18, y=x$ or $x=0$. However, $x \neq 0$. Therefore, $x=y$.

$(\Leftarrow)$ Assume that $x, y \in H, x \ll y$. If $y=0$, then $x \ll y=0$, we can obtain $x=y=0$. If $x \neq 0, y \neq 0$, there is $x=y$ by condition. Therefore, $y$ is a quasi-minimal element of $H$. Thus, $H$ is a QM-hyper BCI algebra.

Theorem 12. If $(H, \circ)$ is a generalized quasi-left alter-hyper BCI-algebra, then it is QM-hyper BCI-algebra.

Proof. Assume that $(H, \circ)$ is a generalized quasi-left alter-hyper $B C I$-algebra. According to Theorem 10, $H$ is a generalized quasi-left alter $B C I$-algebra. Let $B(H)$ be $B C K$-part of $H$, and $G(H)$ be $B C K$-remainder of $H$. Then, for any $x, y \in H$, assume that $x \ll y$ and $x \neq y$. That is,

$$
x=x \circ 0=x \circ(x \circ y)=0 \circ(0 \circ y) .
$$

(1) When $y \in B(H), x=0 \circ(0 \circ y)=0 \circ 0=0$.

(2) When $y \in G(H), x=0 \circ(0 \circ y)=y$, but $x \neq y$. Therefore, $y$ is a quasi-minimal element of $H$. As $y$ is arbitrary, $H$ is QM-hyper BCI algebra.

However, not every QM-hyper BCI algebra is a generalized quasi-left alter-hyper BCI-algebra, see Example 4.

Example 4. Let $H=\{0,1,2,3,4\}$. The operation $\circ$ on $H$ is shown in Table 5. Clearly, $H$ is QM-hyper BCI-algebra.

However, $H$ is not a generalized quasi-left alter-hyper BCI-algebra, since $1 \circ(1 \circ 0)=$ $\{0,1\}, 0 \circ(0 \circ 0)=0,1 \neq 0$.

Above all, we prove that generalized quasi-left alter BCI-algebra, QM-BCI algebra and generaized quasi-left alter hyper $B C I$-algebra are equivalent to one another. Additionally, they are QM-hyper BCI-algebra.

\section{Quasi-Hyper BCI-Algebra}

At the beginning of this part, we introduce the definition of quasi-hyper BCI-algebras.

Definition 20. Let $(H, \circ)$ be a hyper groupoid containing 0 . If it meets the following conditions:

$(Q H C I 1)(x \circ z) \circ(y \circ z) \ll x \circ y, \forall x, y, z \in H$;

$(Q H C I 2)(x \circ y) \circ z=(x \circ z) \circ y, \forall x, y, z \in H$;

(QHCI3) $x \ll x, \forall x \in H$;

$(Q H C I 4) x \ll y$ and $y \ll x \Rightarrow x=y, \forall x, y \in H$

(QHCI5) $x \ll x \circ 0, \forall x \in H$

(QHCI6) $x \ll 0 \Rightarrow x=0, \forall x \in H$.

At that time, it is a quasi-hyper BCI-algebra.

Remark 1. (1) Every weak hyper BCI-algebra is a quasi-hyper BCI-algebra;

(2) Every hyper BCI-algebra is a quasi-hyper BCI-algebra.

In thia sectin, we give some examples of quasi-hyper $B C I$-algebra, and they show that not every quasi-hyper $B C I$-algebra is a (weak) hyper $B C I$-algebra.

Example 5. (1) Assume that $H=\{0,1,2,3,4\}$. The operation on $H$ is defined in Table 7 : 
Table 7. Quasi-hyper BCI algebra.

\begin{tabular}{cccccc}
\hline$\circ$ & $\mathbf{0}$ & $\mathbf{1}$ & $\mathbf{2}$ & $\mathbf{3}$ & $\mathbf{4}$ \\
\hline 0 & $\{0,1,2,3,4\}$ & $\{0,1,2,3,4\}$ & $\{0,1,2,3,4\}$ & $\{0,1,2,3,4\}$ & $\{0,1,2,3,4\}$ \\
1 & $\{1,2,3,4\}$ & $\{0,1,2,3,4\}$ & $\{0,1,2,3,4\}$ & $\{0,1,2,3,4\}$ & $\{0,1,2,3,4\}$ \\
2 & $\{2,3,4\}$ & $\{1,2,3,4\}$ & $\{0,1,2,3,4\}$ & $\{0,1,2,3,4\}$ & $\{0,1,2,3,4\}$ \\
3 & $\{3,4\}$ & $\{2,3,4\}$ & $\{1,2,3,4\}$ & $\{0,1,2,3,4\}$ & $\{0,1,2,3,4\}$ \\
4 & 4 & $\{3,4\}$ & $\{2,3,4\}$ & $\{1,2,3,4\}$ & $\{0,1,2,3,4\}$ \\
\hline
\end{tabular}

Clearly, $(H, \circ, 0)$ is a quasi-hyper BCI-algebra. However, it is not a weak hyper BCI-algebra, since $0 \circ(0 \circ 1)=\{0,1,2,3,4\}$ and $2 \ll 1$ is not true.

(2) Assume that $H=\{0,1,2,3,4\}$. The operation on $H$ is defined in Table 8:

Table 8. Quasi-hyper BCI-algebra.

\begin{tabular}{cccccc}
\hline$\circ$ & $\mathbf{0}$ & $\mathbf{1}$ & $\mathbf{2}$ & $\mathbf{3}$ & $\mathbf{4}$ \\
\hline 0 & $\{0,1,2,3,4\}$ & $\{0,1,2,3,4\}$ & $\{0,1,2,3,4\}$ & $\{0,1,2,3,4\}$ & $\{0,1,2,3,4\}$ \\
1 & $\{1,2,3,4\}$ & $\{0,1,2,3,4\}$ & $\{0,1,2,3,4\}$ & $\{0,1,2,3,4\}$ & $\{0,1,2,3,4\}$ \\
2 & $\{2,3,4\}$ & $\{1,2,3,4\}$ & $\{0,1,2,3,4\}$ & $\{0,1,2,3,4\}$ & $\{0,1,2,3,4\}$ \\
3 & $\{3,4\}$ & $\{1,2,3,4\}$ & $\{1,2,3,4\}$ & $\{0,1,2,3,4\}$ & $\{0,1,2,3,4\}$ \\
4 & 4 & $\{1,2,3,4\}$ & $\{1,2,3,4\}$ & $\{1,2,3,4\}$ & $\{0,1,2,3,4\}$ \\
\hline
\end{tabular}

Clearly, $(H, \circ, 0)$ is a quasi-hyper BCI-algebra. However, it is not a hyper BCI-algebra, since $0 \circ(0 \circ 0)=\{0,1,2,3,4\}$ and $0 \notin 1 \circ 0$.

Proposition 3. In quasi-hyper BCI-algebra $(H, \circ)$, the following holds: $\forall x, y, z \in H$ and, for all non-empty subsets, $A$ and $B$ of $H$,

(1) $0 \circ(x \circ y) \ll y \circ x$,

(2) $A \ll A$,

(3) $A \subseteq B \Rightarrow A \ll B$,

(4) $A \ll 0 \Rightarrow A=0$,

(5) $x \circ y=0 \Rightarrow(x \circ z) \circ(y \circ z)=0$ and $x \circ z \ll y \circ z$,

(6) $A \circ 0=0 \Rightarrow A=0$,

(7) $x \ll y \Rightarrow 0 \ll y \circ x$,

(8) $x \circ x=0 \Rightarrow|y \circ z|=1$.

(9) $x \circ 0 \ll\{y\} \Rightarrow x \ll y$,

(10) $y \ll z \Rightarrow x \circ z \ll x \circ y$.

Proof. (1) By (QHCI1) and (QHCI3), $0 \circ(x \circ y) \subseteq(y \circ y) \circ(x \circ y) \ll y \circ x$. That is, $0 \circ(x \circ y) \ll y \circ x$.

(2) By (QHCI3), for any $x \in A, x \ll x$, that is $0 \in x \circ x$. Then, $A \ll A$.

(3) Let $a \in A$. Then, $a \in B$. By (QHCI3), $x \ll x$ and $0 \in x \circ x$. Then, $A \ll B$.

(4) Let $a \in A$. Then, $a \ll 0$ and so $a=0$. Then, $A=\{0\}$.

(5) By $(\mathrm{QHCI} 1),(x \circ z) \circ(y \circ z) \ll x \circ y=\{0\}$. By (4), $(x \circ z) \circ(y \circ z)=\{0\}$. Therefore, $x \circ z \ll y \circ z$.

(6) Assume that $A \circ 0=0$; then, $A \ll 0$. Therefore, $A=0$.

(7) Assume that $x \ll y$. Then, $0 \in x \circ y$, and so $0 \in 0 \circ 0 \subseteq(y \circ y) \circ(x \circ y) \ll y \circ x$. Hence, $0 \ll y \circ x$.

(8) $\forall x \in H$, let $x \circ x=\{0\}$. Assume that $|y \circ z|>1$; let $m, n \in y \circ z$, and $m \neq n$. Then,

$$
m \circ n \subseteq(y \circ z) \circ(y \circ z) \ll y \circ y=0 \text { and } n \circ m \subseteq(y \circ z) \circ(y \circ z) \ll y \circ y=0
$$

thus, $m \circ n \ll 0, n \circ m \ll 0$ and $m \ll n, n \ll m$. Hence, $m=n$, and so $|y \circ z|=1$.

(9) According to Definition 20,0 $0(x \circ 0) \circ y=(x \circ y) \circ 0$, there exists $p \in x \circ y$ s.t. $0 \in p \circ 0$, that is, $p \ll 0$. By (QHCI6), $p=0$. So $0 \in x \circ y$ and $x \ll y$. 
(10) As $y \ll z$, then $(x \circ z) \circ 0 \subseteq(x \circ z) \circ(y \circ z) \ll x \circ y$. Therefore, $x \circ z \ll x \circ y$.

Proposition 4. In any quasi-hyper BCI-algebra $(H, \circ)$ satisfying $0 \circ 0=0$, the following holds:

(1) $(0 \circ x) \circ(0 \circ x)=0, \forall x \in H$;

(2) $0 \circ x$ is a singleton set, $\forall x \in H$;

(3) $(0 \circ x) \circ 0=0 \circ x, \forall x \in H$.

Proof. (1) $\forall x \in H,(0 \circ x) \circ(0 \circ x) \ll 0 \circ 0=0$. According to Proposition 3(4), $(0 \circ x) \circ$ $(0 \circ x)=0$.

(2) $\forall m, n \in 0 \circ x$, and $m \neq n . m \circ n \subseteq(0 \circ x) \circ(0 \circ x)=0, n \circ m \subseteq(0 \circ x) \circ(0 \circ x)=0$, by (QHCI4), $m \ll n, n \ll m$, then $m=n$. Therefore, $0 \circ x$ is a singleton set.

(3) By (2), let $0 \circ x=m$. By (QHCI5), $m \ll m \circ 0$ and $m \circ m=0$. Assume that $|m \circ 0|>1$, let $a, b \in m \circ 0 . a \circ b \subseteq(m \circ 0) \circ(m \circ 0) \ll m \circ m=0, b \circ a \subseteq(m \circ 0) \circ$ $(m \circ 0) \ll m \circ m=0$, so $a \ll b, b \ll a$. Additionally, $a=b$. Therefore, $|m \circ 0|=1$. As $m \circ 0=(0 \circ x) \circ 0 \subseteq(0 \circ x) \circ(x \circ x) \ll 0 \circ x=m$, and $|m \circ 0|=1, m=m \circ 0$. That is, $(0 \circ x) \circ 0=0 \circ x$.

Proposition 5. In any quasi-hyper $B C I$-algebra $(H, \circ, 0)$, if $\forall x \in H(x \neq 0)$ satisfying $x \circ 0=x$. At that time, it is a weak hyper BCI-algebra. $\forall x \in H$ satisfying $x \circ 0=x$; then, it is a hyper BCI-algebra.

Proof. Firstly, for any $m \in H$ and $m \neq 0, m \circ 0=m$. Then, $0 \circ(0 \circ m) \subseteq(m \circ m) \circ(0 \circ$ $m) \ll(m \circ 0)=m$. Therefore, $(H, \circ, 0)$ is a weak hyper $B C I$-algebra. For any $m \in H$, $0 \circ(0 \circ m) \subseteq(m \circ m) \circ(0 \circ m) \ll(m \circ 0)=x$. So $(H, \circ, 0)$ is a hyper $B C I$-algebra.

Definition 21. A quasi-hyper BCI-algebra $(H, \circ)$ is called standard if $\forall x \in H$, we have $x \circ 0=x$.

Proposition 6. Every standard quasi-hyper BCI-algebra is a hyper BCI-algebra.

Proof. This follows from Proposition 5.

The concept of $H_{v}$-group was introduced by T. Vougiouklis [35]: Let $(H, \cdot)$ be a hyperstructure. If it satisfies: (i) $(x \cdot y) \cdot z \cap x \cdot(y \cdot z) \neq \varnothing, \forall x, y, z \in H$, (ii) $a \cdot H=H \cdot a=H$, $\forall a \in H$, then it is a $H_{v}$-group.

Firstly, define $x \cdot y$ through $x \cdot y=x \circ(0 \circ y), \forall x, y \in H$ in a quasi-hyper BCI-algebra.

Theorem 13. Assume that $(H, \circ)$ is a quasi-hyper BCI-algebra and meets these conditions:

(1) $x \in m \circ(m \circ x), \forall x, m \in H$;

(2) $x \cdot y \cap y \cdot x \neq \varnothing, \forall x, y \in H$;

(3) $x \cdot(y \cdot z)=y \cdot(x \cdot z), \forall x, y, z \in H$. We find that $(H, \cdot)$ is a $H_{v}$-group.

Proof. Obviously, $(x \cdot y) \cdot z=(x \cdot z) \cdot y$ for all $x, y, z \in H$. As

$$
(x \cdot y) \cdot z=(x \circ(0 \circ y)) \circ(0 \circ z)=(x \circ(0 \circ z)) \circ(0 \circ y)=(x \cdot z) \cdot y \text {. }
$$

According to (1), $x \in m \circ(m \circ x) \subseteq m \circ(0 \circ(0 \circ(m \circ x)))=m \cdot 0 \circ(m \circ x) \subseteq m \cdot H$. Hence, $H \subseteq m \cdot H$ and $H=a \cdot H$. Moreover, $x \in m \circ(m \circ x) \subseteq(0 \circ(0 \circ m)) \circ(m \circ x)=$ $(0 \circ(m \circ x)) \circ(0 \circ m)=(0 \circ(m \circ x)) \cdot m \subseteq H \cdot m$. Thus, $x \cdot H=H=H \cdot x$.

According to (2), $x \cdot z \cap z \cdot x \neq \varnothing$, and thus $\exists p \in x \cdot z \cap z \cdot x$. According to (2), there is $q \in p \cdot y \cap y \cdot p$ and thus $q \in p \cdot y \subseteq(x \cdot z) \cdot y=(x \cdot y) \cdot z$. On the other hand, $q \in y \cdot p \subseteq y \cdot(x \cdot z)=x \cdot(y \cdot z)$ by (3). So $q \in(x \cdot y) \cdot z \cap x \cdot(y \cdot z)$.

According to the definition of $H_{v}$-group, we can see that $(H, \cdot)$ is a $H_{v}$-group.

Example 6. Assume that $H=\{0,1,2,3,4\}$ be a quasi-hyper BCI-algebra satisfying those conditions in Theorem 13. Additionally, the operation o on $H$ is shown in Table 9: 
Table 9. Quasi-hyper BCI algebra.

\begin{tabular}{cccccc}
\hline$\circ$ & $\mathbf{0}$ & $\mathbf{1}$ & $\mathbf{2}$ & $\mathbf{3}$ & $\mathbf{4}$ \\
\hline 0 & $\{0,1,2,3,4\}$ & $\{0,1,2,3,4\}$ & $\{0,1,2,3,4\}$ & $\{0,1,2,3,4\}$ & $\{0,1,2,3,4\}$ \\
1 & $\{1,2,3,4\}$ & $\{0,1,2,3,4\}$ & $\{0,1,2,3,4\}$ & $\{0,1,2,3,4\}$ & $\{0,1,2,3,4\}$ \\
2 & $\{2,3,4\}$ & $\{1,2,3,4\}$ & $\{0,1,2,3,4\}$ & $\{0,1,2,3,4\}$ & $\{0,1,2,3,4\}$ \\
3 & $\{3,4\}$ & $\{2,3,4\}$ & $\{1,2,3,4\}$ & $\{0,1,2,3,4\}$ & $\{0,1,2,3,4\}$ \\
4 & 4 & 4 & 4 & 4 & $\{0,1,2,3,4\}$ \\
\hline
\end{tabular}

Then, we obtain a $H_{v}$-group $(H, \cdot)$ and the operation ". " is shown in Table 10:

Table 10. $H_{v}$-group.

\begin{tabular}{cccccc}
\hline$\bullet$ & $\mathbf{0}$ & $\mathbf{1}$ & $\mathbf{2}$ & $\mathbf{3}$ & $\mathbf{4}$ \\
\hline 0 & $\{0,1,2,3,4\}$ & $\{0,1,2,3,4\}$ & $\{0,1,2,3,4\}$ & $\{0,1,2,3,4\}$ & $\{0,1,2,3,4\}$ \\
1 & $\{0,1,2,3,4\}$ & $\{0,1,2,3,4\}$ & $\{0,1,2,3,4\}$ & $\{0,1,2,3,4\}$ & $\{0,1,2,3,4\}$ \\
2 & $\{0,1,2,3,4\}$ & $\{0,1,2,3,4\}$ & $\{0,1,2,3,4\}$ & $\{0,1,2,3,4\}$ & $\{0,1,2,3,4\}$ \\
3 & $\{0,1,2,3,4\}$ & $\{0,1,2,3,4\}$ & $\{0,1,2,3,4\}$ & $\{0,1,2,3,4\}$ & $\{0,1,2,3,4\}$ \\
4 & $\{0,1,2,3,4\}$ & $\{0,1,2,3,4\}$ & $\{0,1,2,3,4\}$ & $\{0,1,2,3,4\}$ & $\{0,1,2,3,4\}$ \\
\hline
\end{tabular}

Theorem 14. Assume that $(H, \circ)$ is a quasi-hyper BCI-algebra and meets those conditions: $\forall x, a, y \in H$,

(1) $x \in a \circ(a \circ x)$,

(2) $x \circ(0 \circ y)=y \circ(0 \circ x)$.

We can see that $(H, \cdot)$ is a hypergroup.

Proof. According to Theorem 13, $x \cdot H=H=H \cdot x$. Morever, $x \cdot y=y \cdot x$ and $(x \cdot y) \cdot z=$ $(x \cdot z) \cdot y$. Then $(x \cdot y) \cdot z=z \cdot(x \cdot y)=z \cdot(y \cdot x)=(y \cdot x) \cdot z=(y \cdot z) \cdot x=x \cdot(y \cdot z)$. So $(H, \cdot)$ is a hypergroup, and is commutative.

Example 7. Let $H=\{0,1,2,3,4\}$ be a quasi-hyper BCI-algebra satisfying the conditions in Theorem 14. The operation on $H$ is shown in Table 11:

Table 11. Quasi-hyper BCI-algebra.

\begin{tabular}{cccccc}
\hline$\circ$ & $\mathbf{0}$ & $\mathbf{1}$ & $\mathbf{2}$ & $\mathbf{3}$ & $\mathbf{4}$ \\
\hline 0 & $\{0,1,2,3,4\}$ & $\{0,1,2,3,4\}$ & $\{0,1,2,3,4\}$ & $\{0,1,2,3,4\}$ & $\{0,1,2,3,4\}$ \\
1 & $\{1,2,3,4\}$ & $\{0,1,2,3,4\}$ & $\{0,1,2,3,4\}$ & $\{0,1,2,3,4\}$ & $\{0,1,2,3,4\}$ \\
2 & $\{2,3,4\}$ & $\{1,3,4\}$ & $\{0,1,2,3,4\}$ & $\{0,1,2,3,4\}$ & $\{0,1,2,3,4\}$ \\
3 & $\{3,4\}$ & $\{2,4\}$ & $\{1,2,4\}$ & $\{0,1,2,3,4\}$ & $\{0,1,2,3,4\}$ \\
4 & 4 & $\{3,4\}$ & $\{2,3,4\}$ & $\{1,2,3,4\}$ & $\{0,1,2,3,4\}$ \\
\hline
\end{tabular}

Then, we obtain a commutative hypergroup $(H, \cdot)$ and the operation "." is shown in Table 12:

Table 12. Hyper group.

\begin{tabular}{cccccc}
\hline$\bullet$ & $\mathbf{0}$ & $\mathbf{1}$ & $\mathbf{2}$ & $\mathbf{3}$ & $\mathbf{4}$ \\
\hline 0 & $\{0,1,2,3,4\}$ & $\{0,1,2,3,4\}$ & $\{0,1,2,3,4\}$ & $\{0,1,2,3,4\}$ & $\{0,1,2,3,4\}$ \\
1 & $\{0,1,2,3,4\}$ & $\{0,1,2,3,4\}$ & $\{0,1,2,3,4\}$ & $\{0,1,2,3,4\}$ & $\{0,1,2,3,4\}$ \\
2 & $\{0,1,2,3,4\}$ & $\{0,1,2,3,4\}$ & $\{0,1,2,3,4\}$ & $\{0,1,2,3,4\}$ & $\{0,1,2,3,4\}$ \\
3 & $\{0,1,2,3,4\}$ & $\{0,1,2,3,4\}$ & $\{0,1,2,3,4\}$ & $\{0,1,2,3,4\}$ & $\{0,1,2,3,4\}$ \\
4 & $\{0,1,2,3,4\}$ & $\{0,1,2,3,4\}$ & $\{0,1,2,3,4\}$ & $\{0,1,2,3,4\}$ & $\{0,1,2,3,4\}$ \\
\hline
\end{tabular}

Theorem 15. Assume that $(H, \circ)$ is a quasi-hyper BCI-algebra. Denote $M(H)$ as a set of finite products $\rho_{a_{1}} \circledast \ldots \circledast \rho_{a_{s}}\left(\forall a_{1}, \ldots, a_{s} \in H\right)$, where $\circledast$ represents the composition operation of mappings. Then, $M(H)$ is a commutative semigroup. 
Proof. According to Theorem 9.

Example 8. Let $H=\{0,1,2,3,4\}$. Define the operation $\circ$ on $H$ in Table 13,

Table 13. Quasi-hyper BCI-algebra.

\begin{tabular}{ccccccc}
\hline$\circ$ & $\mathbf{0}$ & $\mathbf{1}$ & $\mathbf{2}$ & $\mathbf{3}$ & $\mathbf{4}$ \\
\hline 0 & $\{0,1,2,3,4\}$ & $\{0,1,2,3,4\}$ & $\{0,1,2,3,4\}$ & $\{0,1,2,3,4\}$ & $\{0,1,2,3,4\}$ \\
1 & $\{1,2,3,4\}$ & $\{0,1,2,3,4\}$ & $\{0,1,2,3,4\}$ & $\{0,1,2,3,4\}$ & $\{0,1,2,3,4\}$ \\
2 & $\{2,3,4\}$ & $\{2,3,4\}$ & $\{0,1,2,3,4\}$ & $\{0,1,2,3,4\}$ & $\{0,1,2,3,4\}$ \\
3 & $\{3,4\}$ & $\{3,4\}$ & $\{3,4\}$ & $\{0,1,2,3,4\}$ & $\{0,1,2,3,4\}$ \\
4 & 4 & 4 & 4 & $\{3,4\}$ & $\{0,1,2,3,4\}$ \\
\hline
\end{tabular}

Clearly, $(H, \circ)$ is a quasi-hyper BCI-algebra.

That is,

$\rho_{0}: H \rightarrow H: x \mapsto x \circ 0$. So $\rho_{0}=\{\{0,1,2,3,4\},\{1,2,3,4\},\{2,3,4\},\{3,4\}, 4\}$;

$\rho_{1}: H \rightarrow H: x \mapsto x \circ 1$. So $\rho_{1}=\{\{0,1,2,3,4\},\{0,1,2,3,4\},\{2,3,4\},\{3,4\}, 4\}$;

$\rho_{2}: H \rightarrow H: x \mapsto x \circ 2$. So $\rho_{2}=\{\{0,1,2,3,4\},\{0,1,2,3,4\},\{0,1,2,3,4\},\{3,4\}, 4\}$;

$\rho_{3}: H \rightarrow H: x \mapsto x \circ 3$. So $\rho_{3}=\{\{0,1,2,3,4\},\{0,1,2,3,4\},\{0,1,2,3,4\},\{0,1,2,3,4\}, 4\}$;

$\rho_{4}: H \rightarrow H: x \mapsto x \circ 4$.

Therefore, $\rho_{4}=\{\{0,1,2,3,4\},\{0,1,2,3,4\},\{0,1,2,3,4\},\{0,1,2,3,4\},\{0,1,2,3,4\}\}$.

We can verify the following:

$\rho_{0} \circledast \rho_{0}=\rho_{0}, \rho_{0} \circledast \rho_{1}=\rho_{1}, \rho_{0} \circledast \rho_{2}=\rho_{2}, \rho_{0} \circledast \rho_{3}=\rho_{3}, \rho_{0} \circledast \rho_{4}=\rho_{4} ;$

$\rho_{1} \circledast \rho_{0}=\rho_{1}, \rho_{1} \circledast \rho_{1}=\rho_{1}, \rho_{1} \circledast \rho_{2}=\rho_{2}, \rho_{1} \circledast \rho_{3}=\rho_{3}, \rho_{1} \circledast \rho_{4}=\rho_{4} ;$

$\rho_{2} \circledast \rho_{0}=\rho_{2}, \rho_{2} \circledast \rho_{1}=\rho_{2}, \rho_{2} \circledast \rho_{2}=\rho_{2}, \rho_{2} \circledast \rho_{3}=\rho_{3}, \rho_{2} \circledast \rho_{4}=\rho_{4} ;$

$\rho_{3} \circledast \rho_{0}=\rho_{3}, \rho_{3} \circledast \rho_{1}=\rho_{3}, \rho_{3} \circledast \rho_{2}=\rho_{3}, \rho_{3} \circledast \rho_{3}=\rho_{3}, \rho_{3} \circledast \rho_{4}=\rho_{4} ;$

$\rho_{4} \circledast \rho_{0}=\rho_{4}, \rho_{4} \circledast \rho_{1}=\rho_{4}, \rho_{4} \circledast \rho_{2}=\rho_{4}, \rho_{4} \circledast \rho_{3}=\rho_{4}, \rho_{4} \circledast \rho_{4}=\rho_{4}$.

Then, $M(H)=\left\{\rho_{0}, \rho_{1}, \rho_{2}, \rho_{3}, \rho_{4}\right\}$, and $(M(H), \circledast)$ is a commutative semigroup. The operation is shown in Table 14.

Table 14. The adjoint semigroup of quasi-hyper BCI algebra.

\begin{tabular}{cccccc}
\hline$\circledast$ & $\rho_{\mathbf{0}}$ & $\rho_{\mathbf{1}}$ & $\rho_{\mathbf{2}}$ & $\rho_{\mathbf{3}}$ & $\rho_{\mathbf{4}}$ \\
\hline$\rho_{0}$ & $\rho_{0}$ & $\rho_{1}$ & $\rho_{2}$ & $\rho_{3}$ & $\rho_{4}$ \\
$\rho_{1}$ & $\rho_{1}$ & $\rho_{1}$ & $\rho_{2}$ & $\rho_{3}$ & $\rho_{4}$ \\
$\rho_{2}$ & $\rho_{2}$ & $\rho_{2}$ & $\rho_{2}$ & $\rho_{3}$ & $\rho_{4}$ \\
$\rho_{3}$ & $\rho_{3}$ & $\rho_{3}$ & $\rho_{3}$ & $\rho_{3}$ & $\rho_{4}$ \\
$\rho_{4}$ & $\rho_{4}$ & $\rho_{4}$ & $\rho_{4}$ & $\rho_{4}$ & $\rho_{4}$ \\
\hline
\end{tabular}

Definition 22. In quasi-hyper BCI-algebra $(H, 0)$, if $\forall x, y \in H$, and $x \neq y$,

$$
x \circ(x \circ y)=0 \circ(0 \circ y) \text {. }
$$

Then, this is a generalized quasi-left alter quasi-hyper BCI algebra.

Proposition 7. Assume that $(H, O)$ is a generalized quasi-left alter quasi-hyper BCI algebra satisfying $0 \circ 0=0$. Hence, $H$ is a BCI-algebra.

Proof. According to Theorem 10.

Example 9. Assume that $H=\{0,1,2,3,4\}$. The operation on $H$ is defined in Table 15, 
Table 15. Generalized quasi-left alter quasi-hyper $B C I$-algebra.

\begin{tabular}{cccccc}
\hline$\circ$ & $\mathbf{0}$ & $\mathbf{1}$ & $\mathbf{2}$ & $\mathbf{3}$ & $\mathbf{4}$ \\
\hline 0 & $\{0,1,2,3,4\}$ & $\{0,1,2,3,4\}$ & $\{0,1,2,3,4\}$ & $\{0,1,2,3,4\}$ & $\{0,1,2,3,4\}$ \\
1 & $\{1,2,3,4\}$ & $\{0,1,2,3,4\}$ & $\{0,1,2,3,4\}$ & $\{0,1,2,3,4\}$ & $\{0,1,2,3,4\}$ \\
2 & $\{2,3,4\}$ & $\{1,2,3,4\}$ & $\{0,1,2,3,4\}$ & $\{0,1,2,3,4\}$ & $\{0,1,2,3,4\}$ \\
3 & $\{3,4\}$ & $\{1,2,3,4\}$ & $\{1,2,3,4\}$ & $\{0,1,2,3,4\}$ & $\{0,1,2,3,4\}$ \\
4 & 4 & $\{2,3,4\}$ & $\{1,2,3,4\}$ & $\{1,2,3,4\}$ & $\{0,1,2,3,4\}$ \\
\hline
\end{tabular}

Then, $(H, \circ)$ is a generalized quasi-left alter quasi-hyper BCI-algebra.

Definition 23. A quasi-hyper BCI-algebra $(H, \ll, \circ, 0)$ is called a QM-quasi hyper BCI-algebra, if all elements of $\mathrm{H}$ are quasi-minimal.

Theorem 16. Assume that $(H, \ll, 0,0)$ be a quasi-hyper BCI-algebra. This is a QM-quasi hyper $B C I$-algebra if $\forall x, y \in H-\{0\}$,

$$
x \ll y \Rightarrow x=y .
$$

Proof. According to Theorem 11.

Example 10. Let $H=\{0,1,2,3,4\}$. The operation on $H$ is defined in Table 16,

Table 16. Quasi-hyper $B C I$ algebra.

\begin{tabular}{cccccc}
\hline$\circ$ & $\mathbf{0}$ & $\mathbf{1}$ & $\mathbf{2}$ & $\mathbf{3}$ & $\mathbf{4}$ \\
\hline 0 & 0 & 0 & 0 & 4 & 3 \\
1 & 1 & $\{0,1\}$ & 1 & 4 & 3 \\
2 & 2 & 2 & $\{0,2\}$ & 4 & 3 \\
3 & 3 & 3 & 3 & 0 & 4 \\
4 & 4 & 4 & 4 & 3 & 0 \\
\hline
\end{tabular}

Clearly, $(H, \circ)$ is a QM-quasi hyper BCI-algebra. However, it is not a generalized quasi-left alter quasi-hyper $B C I$-algebra, since $1 \circ(1 \circ 0)=\{0,1\}, 0 \circ(0 \circ 0)=0,1 \neq 0$.

According to Definition, we know that both QM-BCI algebra and QM-hyper $B C I$ algebra are QM-quasi hyper $B C I$-algebra, but not every QM-quasi hyper $B C I$-algebra is QM-BCI algebra and QM-hyper BCI-algebra; see Example 11.

Example 11. Let $H=\{0,1,2\}$. The operation on $H$ is defined in Table 17,

Table 17. Quasi-hyper $B C I$ algebra.

\begin{tabular}{cccc}
\hline$\circ$ & $\mathbf{0}$ & $\mathbf{1}$ & $\mathbf{2}$ \\
\hline 0 & $\{0,1\}$ & $\{0,1\}$ & 2 \\
1 & 1 & $\{0,1\}$ & 2 \\
2 & 2 & 2 & $\{0,1\}$ \\
\hline
\end{tabular}

Clearly, $(H, 0)$ is a QM-quasi hyper BCI-algebra. However, it is not QM-hyper BCI algebra, since $0 \circ(0 \circ 0)=\{0,1\}, 1 \ll 0$ is not true.

\section{Discussion}

Firstly, we discuss the adjoint semigroup of a generalized quasi-left alter $B C I$-algebra, which is a commutative Clifford semigroup. Then, we introduced QM-BCI algebra and proved that the generalized quasi-left alter $B C I$-algebra is equivalent to QM-BCI algebra. Furthermore, we proved that the generalized quasi-left alter-hyper $B C I$-algebra is a generalized quasi-left alter $B C I$-algebra. In the last part, we proposed the notion of quasi-hyper 
$B C I$ algebra and discuss its properties. We explored the subalgebras of quasi-hyper $B C I$ algebra and the relationships between quasi-hyper $B C I$ algebra and the hypergroup, $H_{v^{-}}$ group. In general, this paper discusses the relationship between (hyper) logical algebra and classical abstract algebra, and the description of the structure of (hyper) logical algebra is more clear. As a further research topic, we can consider exploring the internal connections between (hyper) BCI-algebras, BI-algebras and CA-semihypergroups (see [36-38]).

Author Contributions: Writing—original draft preparation, Y.D.; writing—review and editing, X.Z. All authors have read and agreed to the published version of the manuscript.

Funding: This research was funded by National Science Foundation of China grant number 62081240416 and Natural Science Foundation of Shaanxi Province grant number 2020JQ-698.

Conflicts of Interest: The authors declare no conflict of interest.

\section{References}

1. Meng, J.; Jun, Y.B. BCK-Algebras; Kyung Moon Sa Co.: Seoul, Korea, 1994.

2. Huang, Y.S. BCI-Algebra; Science Press: Beijing, China, 2006.

3. Iorgulescu, A. Implicative-Groups vs. Groups and Generalizations; Matrix Rom: Bucharest, Romania, 2018.

4. Zhang, X.H. A survey of algebraic structures derived from non-classical logics. J. Sichuan Norm. Univ. (Nat. Sci.) 2019, 42, 1-14. (In Chinese)

5. Song, S.Z.; Khan, M.; Smarandance, F.; Jun, Y.B. Interval neutrosophic sets applied to ideals in BCK/BCI-algebras. Neutrosophic Sets Syst. 2017, 18, 16-26.

6. $\quad \mathrm{Hu}$, Q.P.; Iseki, K. On BCI-algebra satisfying $(x * y) * z=x *(y * z)$. Math. Semior Notes 1980, 8, 553-555.

7. Lei, T.D. Generalized associative BCI-algebra (in Chinese). Pure Appl. Math. 1985, 1, 98-102.

8. Zhang, X.H.; Yang, Y.B. A Kind of BCI-algebra and Its Structure. J. Northwest Norm. Univ. Sci. 1992, $28,27-31$.

9. Jun,Y.B.; Xin, X.L.; Roh, E.H. A class of algebras related to BCI-algebras and semigroups. Soochow J. Math. 1998, $24,309-321$.

10. Huang, W. On BCI-algebras and semigroups. Math. Jpn. 1995, 42, 59-64.

11. Huang, W.P.; Wang, D.J. Adjoint semigroups of BCI-algebra. SEA Bull. Math. 1995, 19, 95-98.

12. Zhang, X.H.; Ye, R.F. BZ-algebra and group. J. Math. Phys. Sci. 1995, 29, 223-233.

13. Marty, F. Sur une generalization de la notion de groupe. Sartryck ur Forhandlingar via Altonde Skandinavioka Matematiker kongressen i Stockholm. 1934, 45-49.

14. Wall, H.S. Hypergroups. Am. J. Math. 1937, 59, 77-98. [CrossRef]

15. Corsini, P.; Leoreanu, V. Applications of hyperstructure theory. In Advances in Mathematics; Kluwer Academic Publishers: Dordrecht, The Netherlands, 2003.

16. Davvaz, B.; Nezhad, A.D.; Heidari, M.M. Inheritance examples of algebraic hyperstructures. Inf. Sci. 2013, $224,180-187$. [CrossRef]

17. Davvaz, B. Semihypergroup Theory; Elsevier: Amsterdam, The Netherlands, 2016.

18. Jun, Y.B.; Zahedi, M.M.; Xin, X.L.; Borzooei, R.A. On hyper BCK-algebras. Ital. J. Pure Appl. Math. 2000, 8, $127-136$.

19. Jun, Y.B.; Xin, X.L. Scalar elements and hyperatoms of hyper BCK-algebras. Sci. Math. 1999, 2, 303-309.

20. Jun, Y.B.; Song, S.Z.; Shim, W.H. On implicative hyper K-ideals of hyper K-algebras. Sci. Math. Jpn. 2004, 59, 443-450.

21. Jun, Y.B.; Roh, E.H. Fuzzy (weak) implicative hyper K-ideals. Bull. Korean Math. Soc. 2006, 43, 141-148. [CrossRef]

22. Jun, Y.B.; Kang, M.S.; Kim, H.S. Hyper MV-deductive systems of hyper MV-algebras. Commun. Korean Math. Soc. 2010, 25, 537-545. [CrossRef]

23. Jun, Y.B.; Song, S.-Z. Crossing cubic ideals of BCK/BCI-algebras. J. Algebr. Hyperstruct. Log. Algebr. 2011, 2, 17-31. [CrossRef]

24. Jun, Y.B.; Roh, E.H.; Harizavi H. Hyper BCC-algebras. Honam Math. J. 2006, 28, 57-67.

25. Borzooei, R.A.; Dudek W.A.; Koohestanki N. On hyper BCC-algebras. Int. J. Math. Math. Sci. 2006, 2006, 049703, http:/ /dx.doi.org/10.1155/IJMMS/2006/49703. [CrossRef]

26. Xin, X.L. Hyper BCI-algebras. Discuss. Math. Gen. Algebra Appl. 2006, 26, 5-19. [CrossRef]

27. Ahadpanah, A.; Saeid, A.B. Smarandache hyper BCC-algebra. Comput. Math. Appl. 2011, 61, 2490-2497. [CrossRef]

28. Borzooei, R.A.; Saar, B.G.; Ameri, R. On hyper EQ-algebras. Ital. J. Pure Appl. Math. 2013, 31, 77-96.

29. Mao, X.Y.; Zhou, H.J. Classification of proper hyper BCI-algebras of order 3. Appl. Math. Inf. Sci. 2015, 9, 387-393. [CrossRef]

30. Jun, Y.B. Multipolar fuzzy hyper BCK-ideals of hyper BCK-algebras. J. Algebr. Hyperstruct. Log. Algebr. 2020, 1, 37-47. [CrossRef]

31. Borzooei, R.A.; Kologani, M.A. An overview of hyper logical algebras. J. Algebr. Hyperstruct. Log. Algebr. 2020, 1, 31-50. [CrossRef]

32. Du, Y.D.; Zhang, X.H. Hyper BZ-algebras and semigroups. J. Algebr. Hyperstruct. Log. Algebr. 2021, 2, 13-28. [CrossRef]

33. Howie, J.M. Fundamentals of Semigroup Theory; Clarendon Press: Oxford, UK, 1995; pp. 103-107.

34. Yang, W.Q. BCI-Algebras and Semigroups, 1th ed.; Science Press: Beijing, China, 2011; p. 75. (In Chinese)

35. Vougiouklis, T. A new class of hyperstructures. J. Comb. Inf. Syst. Sci. 1995, 20, 229-239.

36. Zhang, X.; Borzooei, R.; Jun, Y. Q-filters of quantum B-algebras and basic implication algebras. Symmetry 2018, 10, 573. [CrossRef] 
37. Zhang, X.; Ma, X.; Wang, X. Filters in strong BI-algebras and residuated pseudo-SBI-algebras. Mathematics 2020, 8, 1513. [CrossRef]

38. Hu, M.H.; Zhang, X.H. On Cyclic Associative Semihypergroups and Neutrosophic Extended Triplet Cyclic Associative Semihypergroups. Mathematics 2022, 10, 535. [CrossRef] 